\title{
High-Order Output-Based Adaptive Simulations of Turbulent Flow in Two Dimensions
}

\author{
Marco A. Ceze* and Krzysztof J. Fidkowski ${ }^{\dagger}$ \\ Department of Aerospace Engineering, University of Michigan, Ann Arbor, MI 48109, USA
}

\begin{abstract}
We present output-based high-order adaptive results for several benchmark twodimensional turbulent-flow simulations. The discretization is a high-order discontinuous Galerkin (DG) finite element method, and the equations solved are compressible Navier-Stokes, Reynolds-averaged with a modified version of the SpalartAllmaras (SA) one-equation model. We study mesh refinement requirements through automated output-based adaptation in which a discrete adjoint solution associated with an output, e.g. the drag coefficient, weights a fine-space residual and automatically selects the elements that need more resolution. The roles of high-order and mesh anisotropy are also investigated. Finally, we investigate differences between two mesh refinement strategies: hanging-node refinement of structured meshes versus metric-based remeshing of unstructured triangles.
\end{abstract}

\section{Introduction}

Although improvements in computing capabilities have made advanced computational fluid dynamics techniques such as large-eddy simulation (LES) possible for a range of applications, the Reynolds-averaged Navier-Stokes equations remain an invaluable tool routinely used in analysis and design. Compared to LES, RANS simulations are much cheaper because they can take advantage of anisotropic (stretched) computational elements that reduce the degrees-of-freedom required to accurately resolve thin boundary and shear layers. This advantage is not always easy to realize, in particular for high-order methods that require curved elements, which are difficult to keep from tangling/inverting when stretched.

High-order methods for RANS suffer from additional debate and scrutiny: RANS solutions often possess singular features that do not lend themselves to high-order approximation, and RANS modeling errors are generally viewed as dominant compared to numerical resolution (discretization) errors that high-order would address. Regarding the latter point, our position is that both modeling and numerical errors need to be estimated and controlled through methods appropriate for each error. For instance, modeling errors may be addressed through an uncertainty quantification study, and this study may require simulations with different model parameter settings but low discretization errors to isolate the effects of the parameters on the model.

Regarding the former point of RANS solutions containing singular features, these features can be isolated with a proper mesh adaptation technique: most flowfields will still possess large smooth regions where high-order will be advantageous. ${ }^{1}$ In particular, output-based methods ${ }^{2-5}$ offer a systematic approach for identifying regions of the domain that require more resolution for the prediction of scalar outputs of interest. These methods also return error estimates that can improve

${ }^{*}$ Postdoctoral Associate, AIAA Member

${ }^{\dagger}$ Associate Professor, AIAA Senior Member 
robustness of solution verification and uncertainty quantification studies. It is for these reasons that we consider output-based methods in the present study.

In this paper, we apply a high-order adaptive solution technique to several test cases modeled with the two-dimensional Reynolds-averaged Navier-Stokes (RANS) equations, closed with a recent modification of the Spalart-Allmaras (SA) one-equation model. ${ }^{6}$ Many previous works have investigated the RANS-SA equations, including in a high-order adaptive setting. ${ }^{1,7-10}$ The majority of the latter work has focused on demonstrating benefits of adaptive refinement and/or high-order over uniform or heuristic refinement for such flows. These comparisons have been done in solely structured and solely unstructured settings. The present work distinguishes itself in that we compare both structured and unstructured mesh refinement techniques, and that we consider a set of well-defined benchmark test cases with available previous (typically second-order) data.

The remainder of this paper is organized as follows. Section II presents the RANS-SA equations, and Section III discusses their discretization. Sections IV and V describe the output error estimation and adaptation techniques, and Section VI presents results for the several benchmark cases considered. Section VII concludes with a summary and a discussion of possible future directions.

\section{Turbulence Model}

We use the Spalart-Allmaras turbulence model, modified for stability for negative values of the turbulence working variable, $\tilde{\nu}^{6}$ The Reynolds-averaged Navier-Stokes (RANS) equations closed with this turbulence model read

$$
\begin{array}{ll}
\partial_{t} \rho+\partial_{j}\left(\rho u_{j}\right) & =0 \\
\partial_{t}\left(\rho u_{i}\right)+\partial_{j}\left(\rho u_{j} u_{i}+p \delta_{i j}\right) & =\partial_{j} \tau_{i j} \\
\partial_{t}(\rho E)+\partial_{j}\left(\rho u_{j} H\right) & =\partial_{j}\left(u_{i} \tau_{i j}-q_{j}\right) \\
\partial_{t}(\rho \tilde{\nu})+\partial_{j}\left(\rho u_{j} \tilde{\nu}\right) & =\frac{1}{\sigma} \partial_{j}\left[\left(\mu+\rho \tilde{\nu} f_{n}\right) \partial_{j} \tilde{\nu}\right]+\frac{c_{b 2} \rho}{\sigma} \partial_{j} \tilde{\nu} \partial_{j} \tilde{\nu}+P-D
\end{array}
$$

where $\rho$ is the density, $\rho u_{j}$ is the momentum, $E$ is the total energy, $H=E+\frac{p}{\rho}$ is the total enthalpy,

$p=(\gamma-1)\left(\rho E-\frac{1}{2} \rho u_{k} u_{k}\right)$ is the pressure, $\gamma$ is the ratio of specific heats, and $i, j$ index the spatial dimension, dim. The Reynolds stress, $\tau_{i j}$, is

$$
\tau_{i j}=2\left(\mu+\mu_{t}\right) \bar{\epsilon}_{i j}, \quad \bar{\epsilon}_{i j}=\frac{1}{2}\left(\partial_{i} u_{j}+\partial_{j} u_{i}\right)-\frac{1}{3} \partial_{k} u_{k} \delta_{i j}
$$

$\mu$ is the laminar dynamic viscosity, obtained using Sutherland's law,

$$
\mu=\mu_{\mathrm{ref}}\left(\frac{T}{T_{\mathrm{ref}}}\right)^{1.5}\left(\frac{T_{\mathrm{ref}}+T_{\mathrm{s}}}{T+T_{\mathrm{s}}}\right),
$$

where $T$ is the temperature, and the eddy viscosity, $\mu_{t}$, is

$$
\mu_{t}=\left\{\begin{array}{ll}
\rho \tilde{\nu} f_{v 1} & \tilde{\nu} \geq 0 \\
0 & \tilde{\nu}<0
\end{array} \quad f_{v 1}=\frac{\chi^{3}}{\chi^{3}+c_{v 1}^{3}}, \quad \chi=\frac{\tilde{\nu}}{\nu} .\right.
$$

The heat flux, $q_{j}$, is given by

$$
q_{j}=\left(k+k_{t}\right) \partial_{i} T, \quad k=C_{p} \mu / P r, \quad k_{t}=C_{p} \mu_{t} / P r_{t}
$$

The production term, $P$, is

$$
P=\left\{\begin{array}{ll}
c_{b 1} \tilde{S} \rho \tilde{\nu} & \chi \geq 0 \\
c_{b 1} S \rho \tilde{\nu} & \chi<0
\end{array},\right.
$$


where the modified vorticity $\tilde{S}$ is written as

$$
\tilde{S}=\left\{\begin{array}{ll}
S+\bar{S} & \bar{S} \geq-c_{v 2} S \\
S+\frac{S\left(c_{v 2}^{2} S+c_{v 3} \bar{S}\right)}{\left(c_{v 3}-2 c_{v 2}\right) S-\bar{S}} & \bar{S}<-c_{v 2} S
\end{array} \quad, \quad \bar{S}=\frac{\tilde{\nu} f_{v 2}}{\kappa^{2} d^{2}}, \quad f_{v 2}=1-\frac{\chi}{1+\chi f_{v 1}} .\right.
$$

In Equation 3, $S=\sqrt{2 \Omega_{i j} \Omega_{i j}}$ is the vorticity magnitude (summation implied on $i, j$ ), and $\Omega_{i j}=$ $\frac{1}{2}\left(\partial_{i} v_{j}-\partial_{j} v_{i}\right)$ is the vorticity tensor. $d$ is the distance to the closest wall. The destruction term, $D$, is given by

$$
D=\left\{\begin{array}{cc}
c_{w 1} f_{w} \frac{\rho \tilde{\nu}^{2}}{d^{2}} & \chi \geq 0 \\
-c_{w 1} \frac{\rho \tilde{\nu}^{2}}{d^{2}} & \chi<0
\end{array}, \quad f_{w}=g\left(\frac{1+c_{w 3}^{6}}{g^{6}+c_{w 3}^{6}}\right)^{1 / 6}, \quad g=r+c_{w 2}\left(r^{6}-r\right), \quad r=\frac{\tilde{\nu}}{\tilde{S} \kappa^{2} d^{2}}\right.
$$

Finally, the coefficient $f_{n}$ in Eqn. 1 is 1 for positive $\tilde{\nu}$ and

$$
f_{n}=\frac{c_{n 1}+\chi^{3}}{c_{n 1}-\chi^{3}}, \quad \text { when } \chi<0 .
$$

Relevant closure coefficients are

$$
\begin{aligned}
& c_{b 1}=0.1355 \\
& c_{w 1}=\frac{c_{b 1}}{\kappa^{2}}+\frac{1+c_{b 2}}{\sigma} \\
& c_{b 2}=0.622 \\
& c_{w 2}=0.3 \\
& c_{v 1}=7.1 \\
& \sigma=2 / 3 \\
& c_{w 3}=2 \\
& \kappa=0.41 \\
& c_{n 1}=16 \\
& c_{v 2}=0.7 \\
& \operatorname{Pr}_{t}=0.9 \\
& c_{v 3}=0.9
\end{aligned}
$$

\section{Discretization}

We discretize Eqn. 1 using a discontinuous Galerkin (DG) finite element method. ${ }^{9,11}$ Defining the state vector as $\mathbf{u}=\left[\rho, \rho u_{i}, \rho E, \rho \tilde{\nu}\right]^{T}$, we write Eqn. 1 in compact conservative form,

$$
\partial_{t} \mathbf{u}+\nabla \cdot \overrightarrow{\mathbf{F}}(\mathbf{u}, \nabla \mathbf{u})+\mathbf{S}(\mathbf{u}, \nabla \mathbf{u})=\mathbf{0}
$$

where $\overrightarrow{\mathbf{F}}$ is the combined inviscid/viscous flux vector, and $\mathbf{S}$ is the source term associated with the turbulence closure equation. We approximate the state as $\mathbf{u}_{h} \in \mathcal{V}_{h}$, where $\mathcal{V}_{h}$ is the space of element-wise discontinuous polynomials of order $p^{\text {a }}$. Multiplying Eqn. 5 by test functions $\mathbf{v}_{h} \in \mathcal{V}_{h}$, integrating by parts on each element, and using the Roe ${ }^{12}$ convective flux and the second form of Bassi and Rebay (BR2) ${ }^{13}$ for the viscous treatment, we obtain the following semilinear form:

$$
\mathcal{R}_{h}\left(\mathbf{u}_{h}, \mathbf{v}_{h}\right)=0 .
$$

Note in Eqn. 1 and Eqn. 5 that the RANS source term depends on the gradient of the state. For the present work we use an adjoint-inconsistent treatment in which the gradient is taken pointwise directly from the polynomial solution approximation, without consideration of interface jump contributions. ${ }^{14}$ Much of the DG discretization is standard; the sections below outline a few practical details.

${ }^{a}$ this order may change from element to element in $p$-refinement 


\section{III.A. Wall distance calculation}

The wall distance, $d(\vec{x})$, required in the SA model is approximated on each element by a polynomial of the same order, $p$, as the solution. The procedure for calculating $d(\vec{x})$ is brute force: Lagrange interpolating polynomials are used, and at each node associated with a Lagrange polynomial in every element, the wall distance is calculated. This calculation considers all of the boundary faces associated with the walls in the domain. The distance to each boundary node is calculated to preselect the closest boundary faces. For each of these boundary faces, which are high-order/curved, the wall distance is estimated by calculating the minimum distances to a set of facets obtained by subdividing the high-order face into $2(Q+1)$ linear segments. Although this distance function calculation could be made more efficient, its cost is negligible compared to the flow solution.

\section{III.B. Symmetry boundary conditions}

Several test cases in the results call for symmetry boundary conditions. In the continuous limit, symmetry requires vanishing normal state derivatives. A finite-dimensional solution will generally violate these requirements pointwise, so that we enforce the BCs weakly. This enforcement involves transforming the state and gradient, similarly to methods in previous works, ${ }^{15}$ though we construct a state/gradient on the boundary instead of employing a ghost cell. Starting with the state, we require that at a symmetry boundary all vectors in the state (e.g. a velocity) have their normal components zeroed out. This results in a linear transformation from the interior $\left(\mathbf{u}^{+}\right)$to the boundary $\left(\mathbf{u}^{b}\right)$ state vector, which reads $\mathbf{u}^{b}=\mathbf{A} \mathbf{u}^{+}$. $\mathbf{A}$ is the identity matrix for all states except the momentum, which transforms as $(\rho \vec{v})^{b}=\underline{V}(\rho \vec{v})^{+}$, where $\underline{V}=\underline{I}-\vec{n} \otimes \vec{n}=\delta_{i j}-n_{i} n_{j}$. $\vec{n}$ is the outward-pointing normal, and $\underline{I}=\delta_{i j}$ is the $\operatorname{dim} \times \operatorname{dim}$ identity matrix.

The state gradient transformation must account for possibly nonzero normal velocity components. We first consider a hypothetical ghost state $\left(\mathbf{u}^{-}\right)$and gradient $\left(\nabla \mathbf{u}^{-}\right)$, obtained by reflecting the velocity about the symmetry line. Specifically, $\mathbf{u}^{-}=\mathbf{B u}^{+}$, where $\mathbf{B}$ is an identity matrix for all states except the momentum, which transforms as $(\rho \vec{v})^{-}=\underline{W}(\rho \vec{v})^{+}$, where $\underline{W}=\underline{I}-2 \vec{n} \otimes \vec{n}=\delta_{i j}-2 n_{i} n_{j}$. Note that $\mathbf{B}=2 \mathbf{A}-\mathbf{I}$ and that $\underline{W}=2 \underline{V}-\underline{I}$. Differentiating the expression for $\mathbf{u}^{-}$in space gives the gradient, which we must reflect by applying $\underline{W}$, so that $\nabla \mathbf{u}^{-}=\mathbf{B} \nabla \mathbf{u}^{+} \underline{W}^{T}$. Finally, we obtain the gradient at the boundary, $\nabla \mathbf{u}^{b}$, by averaging the interior and exterior gradients - this is consistent with what would happen in the viscous flux calculation if there were actually a symmetrical mesh on the other side of the symmetry line. So we have

$$
\begin{aligned}
\nabla \mathbf{u}^{b} & =\frac{1}{2}\left(\nabla \mathbf{u}^{+}+\nabla \mathbf{u}^{-}\right)=\frac{1}{2}\left(\nabla \mathbf{u}^{+}+\mathbf{B} \nabla \mathbf{u}^{+} \underline{W}^{T}\right)=\frac{1}{2}\left(\nabla \mathbf{u}^{+}+(2 \mathbf{A}-\mathbf{I}) \nabla \mathbf{u}^{+}\left(2 \underline{V}^{T}-\underline{I}\right)\right) \\
& =\nabla \mathbf{u}^{+}+\mathbf{A} \nabla \mathbf{u}^{+}\left(2 \underline{V}^{T}-\underline{I}\right)-\nabla \mathbf{u}^{+} \underline{V}=\nabla \mathbf{u}^{+} \vec{n} \otimes \vec{n}+\mathbf{A} \nabla \mathbf{u}^{+}(\underline{I}-2 \vec{n} \otimes \vec{n}) .
\end{aligned}
$$

\section{III.C. Scaling of $\tilde{\nu}$}

The SA working variable, $\tilde{\nu}$, will generally be orders of magnitude smaller than the other state components. Scaling or "non-dimensionalization" of $\tilde{\nu}$ is used to make the stored values of $\tilde{\nu}$ closer to the other state components, which helps during the discrete linear solves and when using finite residual convergence tolerances. Instead of $\rho \tilde{\nu}$ we store $\rho \tilde{\nu}^{\prime}$, given by

$$
\rho \tilde{\nu}^{\prime}=\frac{\rho \tilde{\nu}}{\kappa_{S A} \mu_{\infty}}
$$

where $\kappa_{S A}$ is a scaling factor, typically $\mathcal{O}(\sqrt{R e})$, and $\mu_{\infty}$ is the free-stream laminar dynamic viscosity. In addition, the $\mathrm{SA} \tilde{\nu}$ equation is divided by $\kappa_{S A} \mu_{\infty}$. 


\section{III.D. Implicit Solver}

The system of nonlinear equations that forms the primal problem is solved using Newton's method with pseudo-transient continuation ${ }^{16}$ for improved robustness. Two versions of the solver are considered: (1) A relatively aggressive Courant-Friedrichs-Lewy (CFL) number evolution strategy in which the CFL grows by a factor of 2 after each full Newton update, in combination with incomplete Newton updates when certain physical quantities (e.g. density and pressure) change too drastically; and (2) a more moderate CFL evolution strategy in which the increase factor is 1.2 , but in which the physical quantity changes are not limited; rather the line search prevents the residual from growing too quickly and no update is taken if physical constraints are violated. Both strategies are found to perform similarly for the present test cases. The linear systems at each Newton iteration are solved with an element-line preconditioned ${ }^{17}$ GMRES solver.

\section{Output Error Estimation}

Choosing a basis for the test space in Eqn. 6 gives a discrete system of nonlinear equations,

$$
\mathbf{R}(\mathbf{U})=\mathbf{0}
$$

where $\mathbf{U}$ and $\mathbf{R}$, both in $\mathbb{R}^{N}$ are, respectively, the state and residual vectors. For a scalar output, $J(\mathbf{U})$, we define the discrete adjoint vector, $\Psi \in \mathbb{R}^{N}$, as the sensitivity of $J$ to perturbations in R. ${ }^{5}$ The adjoint satisfies the following linear equation,

$$
\left(\frac{\partial \mathbf{R}}{\partial \mathbf{U}}\right)^{T} \boldsymbol{\Psi}+\left(\frac{\partial J}{\partial \mathbf{U}}\right)^{T}=\mathbf{0} .
$$

We use the adjoint to estimate the error in an output when computing on a finite-dimensional approximation space. Without access to infinite resolution, estimating the true numerical error in an output is practically out of reach for general nonlinear problems. We thus restrict ourselves to estimating the output error between two finite-dimensional spaces: a coarse approximation space $\left(\mathcal{V}_{H}\right)$ on which we calculate the state and output, and a fine space $\left(\mathcal{V}_{h}\right.$, obtained by incrementing the approximation order by 1) relative to which we estimate the error. We would like to measure the output error in the coarse solution relative to the fine space,

$$
\text { output error: } \delta J \equiv J_{H}\left(\mathbf{U}_{H}\right)-J_{h}\left(\mathbf{U}_{h}\right)
$$

We assume that the fine approximation space contains the coarse approximation space, so that

the following lossless state injection is possible, $\mathbf{U}_{h}^{H} \equiv \mathbf{I}_{h}^{H} \mathbf{U}_{H}$, where $\mathbf{I}_{h}^{H}$ is the coarse-to-fine state injection (prolongation) operator. On the fine space, the exact solution $\mathbf{U}_{h} \in \mathbb{R}^{N_{h}}$ would give us zero fine-space residuals, $\mathbf{R}_{h}\left(\mathbf{U}_{h}\right)=\mathbf{0}$. However, the state injected from the coarse space will generally not be a fine space solution and hence will not give us zero fine-space residuals, $\mathbf{R}_{h}\left(\mathbf{U}_{h}^{H}\right) \neq \mathbf{0}$. Instead, the injected coarse state solves a perturbed fine-space problem, $\mathbf{R}_{h}\left(\mathbf{U}_{h}^{\prime}\right)-\mathbf{R}_{h}\left(\mathbf{U}_{h}^{H}\right)=\mathbf{0}$. As this is just the fine-space problem with a residual perturbation, the fine-space adjoint, $\mathbf{\Psi}_{h}$, tells us to expect an output perturbation given by the inner product between the adjoint and the residual perturbation,

$$
\delta J \approx-\mathbf{\Psi}_{h}^{T} \mathbf{R}_{h}\left(\mathbf{U}_{h}^{H}\right)
$$

This derivation assumes small perturbations in the state when the output or equations are nonlinear. Note that this error estimate does not require the fine-space primal solution, $\mathbf{U}_{h}$. 


\section{Mesh Adaptation}

The adjoint-weighted residual error estimate in Eqn. 10 can be localized to the elements by keeping track of the contributions from each fine-space element, indexed by $k$ below,

$$
\begin{aligned}
J_{H}\left(\mathbf{U}_{H}\right)-J_{h}\left(\mathbf{U}_{h}\right) \approx-\mathbf{\Psi}_{h}^{T} \mathbf{R}_{h}\left(\mathbf{U}_{h}^{H}\right) & =-\sum_{k} \boldsymbol{\Psi}_{h k}^{T} \mathbf{R}_{h k}\left(\mathbf{U}_{h}^{H}\right) \\
\Rightarrow \epsilon_{k} & \equiv\left|\Psi_{h k}^{T} \mathbf{R}_{h k}\left(\mathbf{U}_{h}^{H}\right)\right|,
\end{aligned}
$$

where the subscript $k$ indicates restriction to element $k$, and the adaptive indicator $\epsilon_{k}$ is obtained by taking the absolute value of the elemental contributions. This indicator then drives mesh adaptation, the goal of which is to reduce the output error. We consider two adaptation strategies, as outlined below.

\section{V.A. Hanging-Node Quadrilateral Refinement}

The first adaptation strategy used in this work is hanging-node refinement of an initially structured quadrilateral mesh. ${ }^{9,11,18}$ In this strategy, a fixed fraction, $f^{\text {frac }}$, of elements with the highest error indicators is flagged for refinement. For the present results, we only consider isotropic refinement in which each quadrilateral is subdivided uniformly into four quadrilaterals, as illustrated in Figure 1. This refinement is done in each element's reference space by employing the referenceto-global coordinate mapping in calculating the refined elements' geometry node coordinates. The refined elements inherit the same geometry approximation order and quadrature rules as the parent coarse element. When curved boundary representations are employed, new nodes introduced on the boundary are snapped to the geometry - this perturbation is usually very small for high-order curved elements on the boundary, as these already approximate the geometry with high fidelity. Elements created in a hanging-node refinement can be marked for $h$-refinement again in subsequent adaptation iterations. In this case, neighbors will be cut to keep one level of refinement difference between adjacent cells, as illustrated in Figure 1.
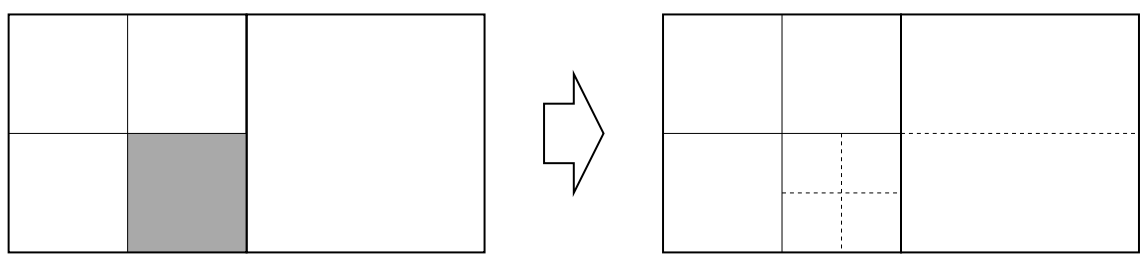

Figure 1. Hanging-node adaptation for a quadrilateral mesh, with a maximum of one level of refinement separating two elements. The shaded element on the left is marked for isotropic refinement, and the dashed lines on the right indicate the additional new edges formed.

\section{V.B. Metric-Based Unstructured Remeshing}

The second adaptation strategy used in this work is an unstructured metric-based remeshing approach on triangles, similar to that presented in previous work. ${ }^{19}$ The idea in this strategy is to, at every adaptation iteration, create a new mesh using the current mesh and the error indicator. During remeshing, the current mesh serves as a background mesh on which the desired metric field is prescribed. The program used for the remeshing is the bi-dimensional anisotropic mesh generator $(\mathrm{BAMG}){ }^{20}$

The first step is a calculation of the current metric field, computed as the grid-implied metric on each element $k$,

$$
\underline{M}_{k}^{c}=\left(\underline{J}_{k} \underline{J}_{k}^{T}\right)^{-1},
$$


where $\underline{J}_{k} \in \mathbb{R}^{\operatorname{dim} \times \operatorname{dim}}$ is the Jacobian of the geometric mapping of the reference element to element $k$. The eigenvalues of the metric are the inverse squares of the principal stretching magnitudes, and the eigenvectors are the principal stretching directions. For curved elements, $\underline{J}_{k}$ is evaluated at the centroid of the element.

We assume a fixed-growth refinement strategy in which the number of elements desired on the refined mesh is $N^{f}=f^{\text {growth }} N^{c}$, where $f^{\text {growth }}>1$ is the growth fraction and $N^{c}$ is the current number of elements. We relate the growth in elements to an error reduction factor through an a priori estimate. In particular, we sum the error indicators on the current mesh to obtain a conservative estimate of the current global error,

$$
e^{c}=\sum_{k} \epsilon_{k}^{c}
$$

Assuming that, with adaptive refinement, the global error decreases at a rate of $r$ (with $h \propto$ $N^{-1 / \operatorname{dim}}$ ), we calculate the global error estimate on the refined mesh as

$$
e^{f}=e^{c}\left(\frac{N^{c}}{N^{f}}\right)^{r / \operatorname{dim}} .
$$

We would like the error to be equidistributed on the fine mesh, which means that every fine space element should have an error of $e^{f} / N^{f}$. We now apply the a priori estimate to each element, and we assume that for anisotropic elements the error depends on the shortest principal length, $h_{1}$. The resulting a priori relationship is

$$
n_{k} \frac{e^{f}}{N^{f}}=\epsilon_{k}^{c}\left(\frac{h_{1}^{f}}{h_{1}^{c}}\right)^{r_{k}}
$$

where $n_{k}$ is the number (not necessarily an integer) of refined elements per current element $k, h_{1}^{f} / h_{1}^{c}$ are the shortest principal lengths on the refined/current meshes, and $r_{k}$ is a possibly element-specific error convergence rate. We estimate the number of fine elements for coarse element $k$ as

$$
n_{k}=\frac{h_{1}^{c}}{h_{1}^{f}} \frac{h_{2}^{c}}{h_{2}^{f}}=\left(\frac{h_{1}^{c}}{h_{1}^{f}}\right)^{2} \frac{A R_{k}^{f}}{A R_{k}^{c}},
$$

where $A R^{f} / A R^{c}$ are the desired/current aspect ratios on element $k$. Substituting Eqn. 15 into Eqn. 14, we obtain an expression for the scaling of the shortest principal stretching length,

$$
\frac{h_{1}^{f}}{h_{1}^{c}}=\left[\frac{e^{f}}{N_{f}} \frac{1}{\epsilon_{k}^{c}} \frac{A R^{c}}{A R^{f}}\right]^{1 /\left(r_{k}+\operatorname{dim}\right)} .
$$

The desired aspect ratio on each element is calculated heuristically from the Hessian (second derivative matrix) of the Mach number scalar field. ${ }^{21,22}$ While only strictly applicable to linear approximations, we have found that the directions obtained from the Hessian correlate well with directions obtained from approaches that use higher-order derivatives. ${ }^{19}$ More sophisticated approaches, such as sampling refinement strategies, ${ }^{10}$ will be investigated in future work.

For the a priori convergence rates in the present work, we use $r=r_{k}=p+1$, where $p$ is the solution approximation order. An exception is "outlier" elements: those whose error indicator $\epsilon_{k}$ is larger than 5 standard deviations from the mean error. On these elements, we assume a first-order rate, $r_{k}=1$. 


\section{Results}

VI.A. Flat Plate, $R e_{L}=5 \times 10^{6},(L=1), M=0.2$

The first case we consider is turbulent flow over a flat plate. The geometry and boundary conditions for this case are set according to NASA's turbulence modeling resource website (Figure 2(a)). The inflow and farfield values for the eddy viscosity are set to 3 times the value of the laminar viscosity, as this value corresponds to a fully turbulent simulation. The laminar viscosity is calculated using Sutherland's law (Eqn. 2) with $T_{s}=110 \mathrm{~K}$ and $T_{\text {ref }}=300 \mathrm{~K}$. Figure 2(b) shows the initial mesh used for this case.

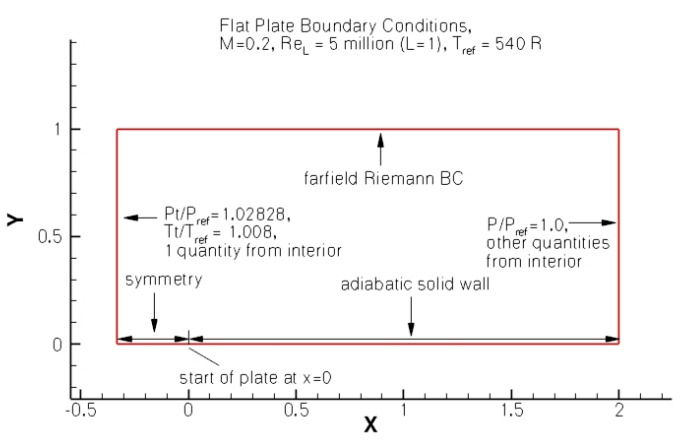

(a) Boundary conditions for the flat plate case

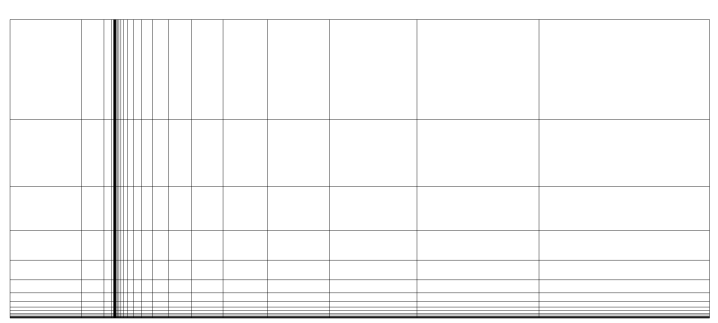

(b) Initial mesh for the flat plate case

Figure 2. Flat plate: initial mesh and boundary conditions.

We initialize the flow with uniform conditions at $M=0.2$ and solve the discretized equations with $p=2$ to a residual tolerance of $10^{-8}$. The output of interest is the total drag on the plate, and at each step of the hanging-node adaptation we select $f^{\text {frac }}=10 \%$ of the elements with the largest error indicators and refine them isotropically. We present a code-to-code comparison in Figure 3(a) of our simulation to the results from CFL3D and FUN3D, provided by NASA's turbulence modeling group. Note that the small difference in the computed drag is due to consistently lower shear stresses shown in Figure 3(b). We observe very good agreement in the turbulent viscosity distribution, Figure 3(c), with just a small discrepancy at the outer edge of the boundary layer.

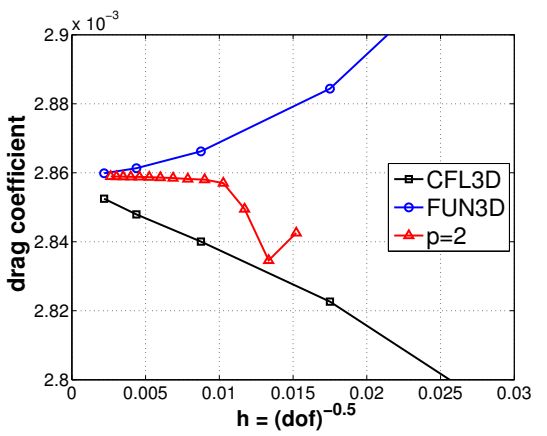

(a) Drag convergence

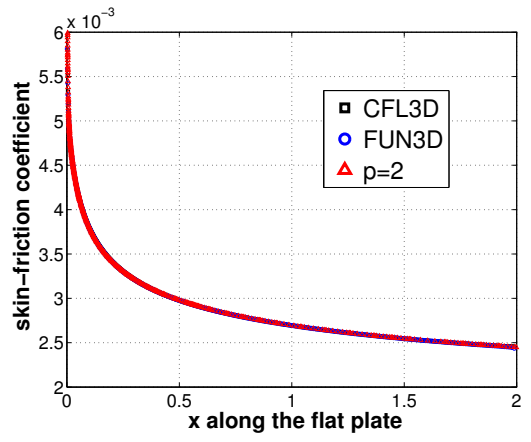

(b) Shear stress coefficient

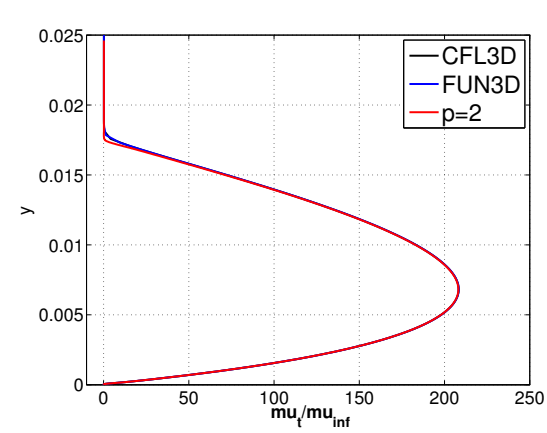

(c) Turbulent/laminar viscosity ratio

Figure 3. Flat plate: drag convergence and comparison of skin-friction coefficient and turbulent viscosity distributions (at $x=0.97$ on the flat plate).

Figure 4 shows the final hanging-node adapted mesh for this case. Note that the adaptive procedure targets the outer edge of the turbulent boundary layer where there is a rapid variation 
of eddy viscosity. The component of the drag adjoint correspondent to the SA variable shows large negative values at the leading edge of the turbulent boundary layer (Figure 4(b)).

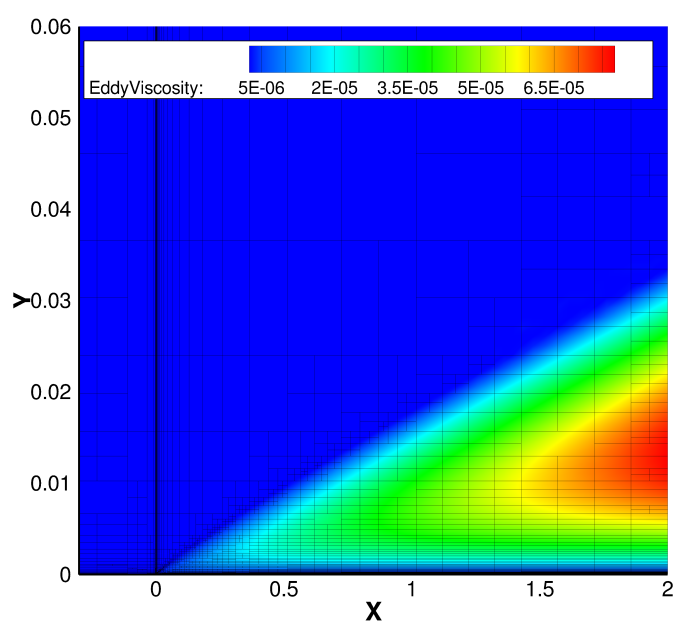

(a) Eddy viscosity contours

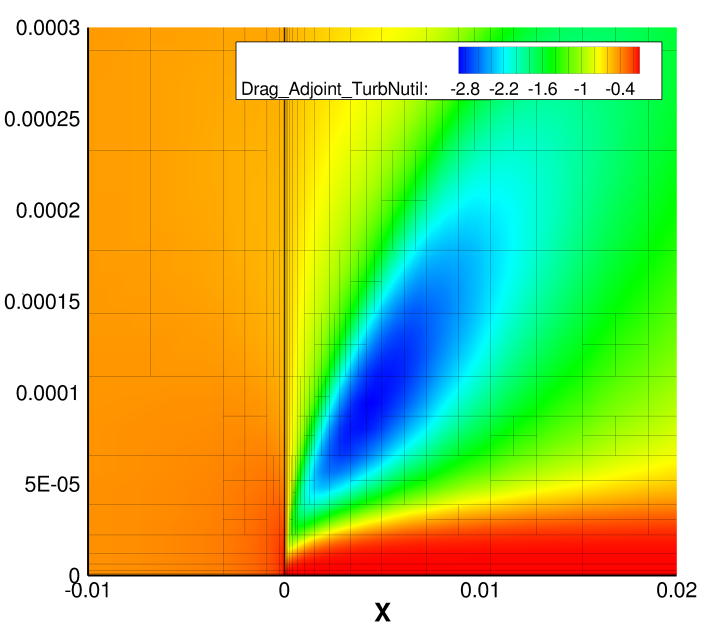

(b) Leading edge zoom

Figure 4. Flat plate: final drag-adapted mesh and field contours of eddy viscosity.

VI.B. Smooth Bump, $R e=3 \times 10^{6}, M=0.2$

This is another verification case from the NASA turbulence modeling resource group. Reynolds number $\operatorname{Re}_{L}=3 \times 10^{6}(L=1)$ flow is simulated in a channel with a bump on the bottom wall. Symmetry boundary conditions are used for the top and bottom of the channel, with the exception of $x \in[0,1.5]$ on the bottom boundary, where an adiabatic wall boundary condition is applied. A static pressure is imposed at the right (outflow) boundary, and total temperature, total pressure, and angle of attack (zero) are prescribed at the inflow. Total/stagnation quantities are computed using a Mach number of $M=0.2$. The dynamic viscosity is computed using Sutherland's law, Eqn. 2, with $T_{\mathrm{s}}=110 \mathrm{~K}$ and $T_{\text {ref }}=300 \mathrm{~K}$. The inflow turbulence eddy viscosity, $\mu_{t}$ is set to 3 times the laminar viscosity.

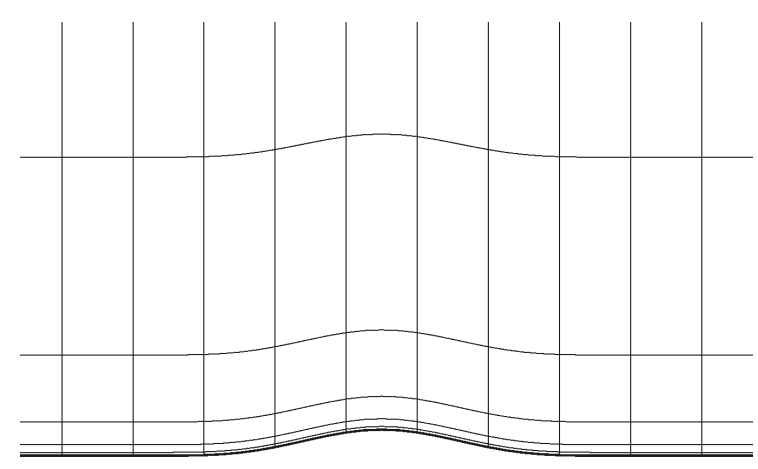

(a) Initial quadrilateral mesh

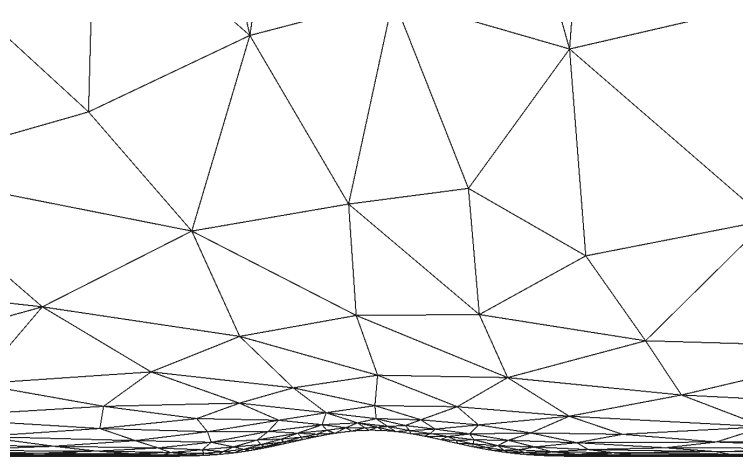

(b) Initial triangular mesh

Figure 5. Smooth bump: Initial meshes for hanging-node (quad) and unstructured (tri) metric-based adaptation.

Initial structured and unstructured meshes for adaptation are shown in Figure 5. Adjoint- 
based adaptive runs are performed from these meshes using the drag force as the target output and adaptive factors of $f^{\text {frac }}=0.07$ and $f^{\text {growth }}=1.3$. Figure 6 shows field plots of the wall distance function and turbulent viscosity on one of the unstructured adapted meshes. Note the heavy refinement in the boundary layer, an area to which the drag output is highly sensitive.

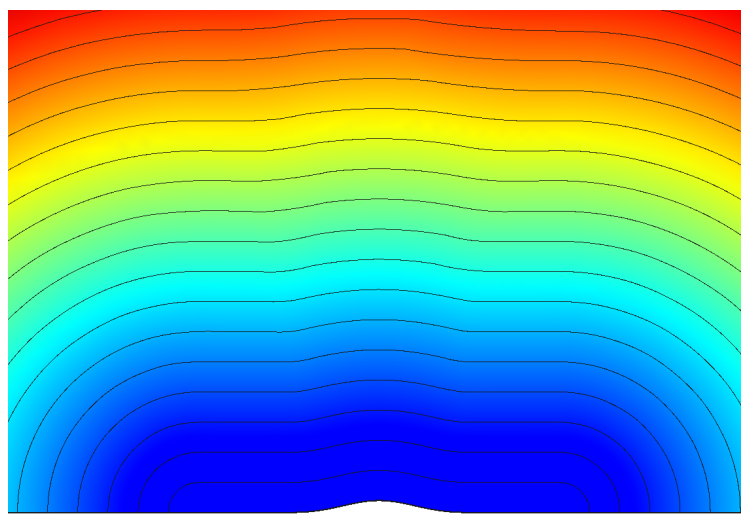

(a) Wall distance

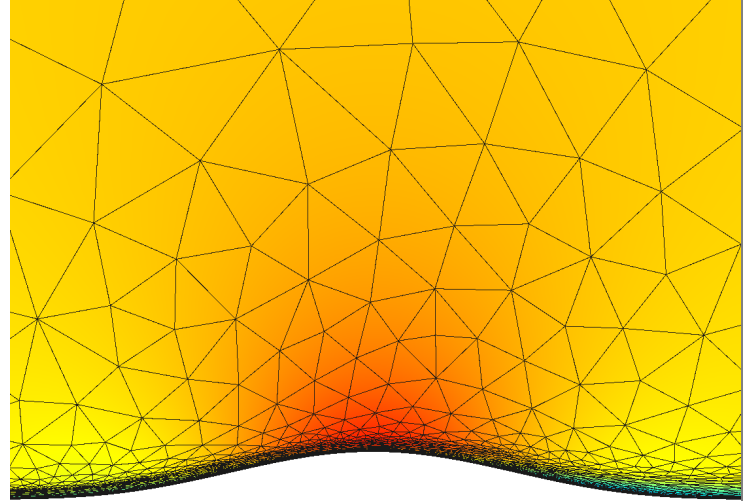

(b) Mach number

Figure 6. Smooth bump: wall distance and Mach number on an adapted mesh with $p=3$ approximation. The Mach number color range is 0 to 0.3 .

As a code-to-code verification of the turbulence model, Figure 7 shows the pressure and skin friction distributions compared to those of two other codes, CFL3D and FUN3D. Data for these codes were obtained from the NASA turbulence modeling resource group. The agreement in both of these quantities is very good - note that flow singularities at the leading and trailing edges of the bump cause oscillations there.

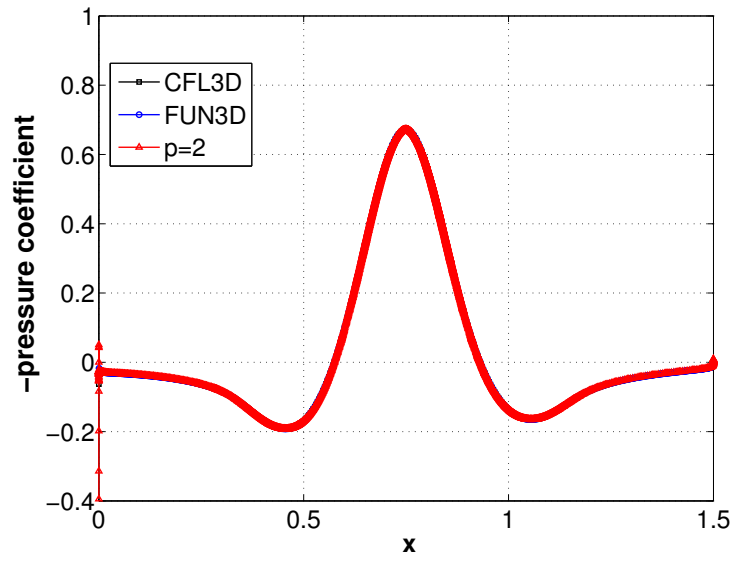

(a) Pressure coefficient

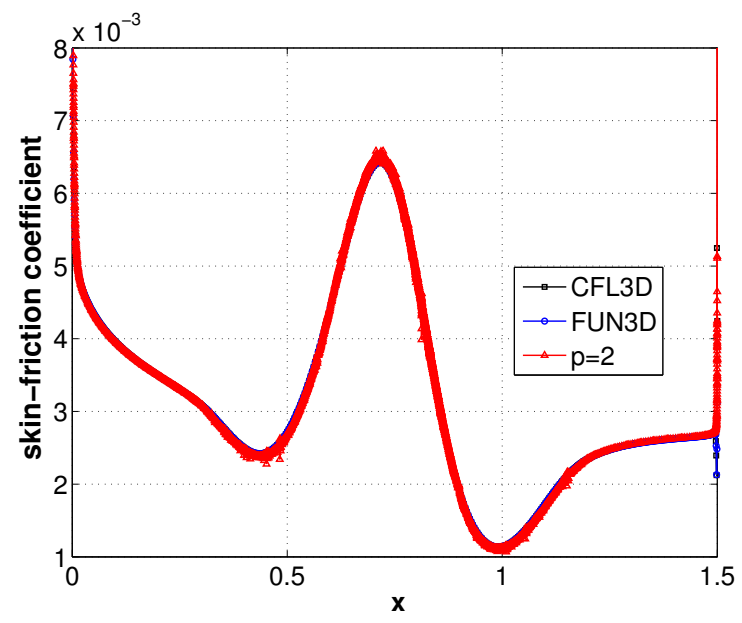

(b) Skin-friction coefficient

Figure 7. Smooth bump: pressure and skin-friction coefficients for the final $p=2$ drag-adapted triangular mesh. Note sporadic pressure and skin friction spikes at the leading and trailing edges of the bump: the flow exhibits singular behavior in these locations.

Figure 8 shows the convergence of the drag and lift coefficients with adaptive mesh refinement at $p=2$ solution approximation. In these plots, the degrees of freedom are measured as dof $=N_{e} n(p)$, where $N_{e}$ is the number of elements and $n(p)$ is the number of unknowns per element: $n(p)=(p+1)^{2}$ for tensor-product approximation and $n(p)=(p+1)(p+2) / 2$ for full-order approximation. Note the rapid convergence of the drag and lift coefficients to their nearly asymptotic values, relative to the second-order codes. The unstructured adaptive results show larger errors on the initial meshes 
because these are relatively under-resolved in the critical boundary layer region. However, after a few adaptive iterations, the drag and lift "snap" to their asymptotic values.

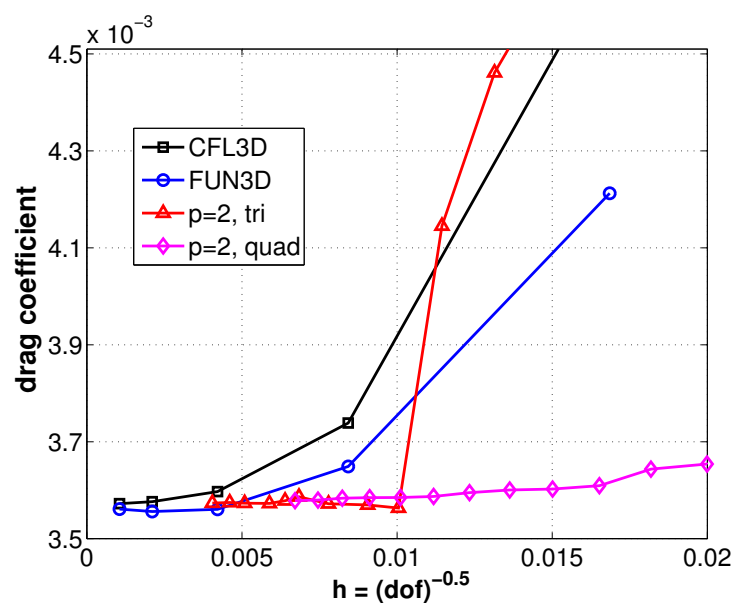

(a) Drag coefficient

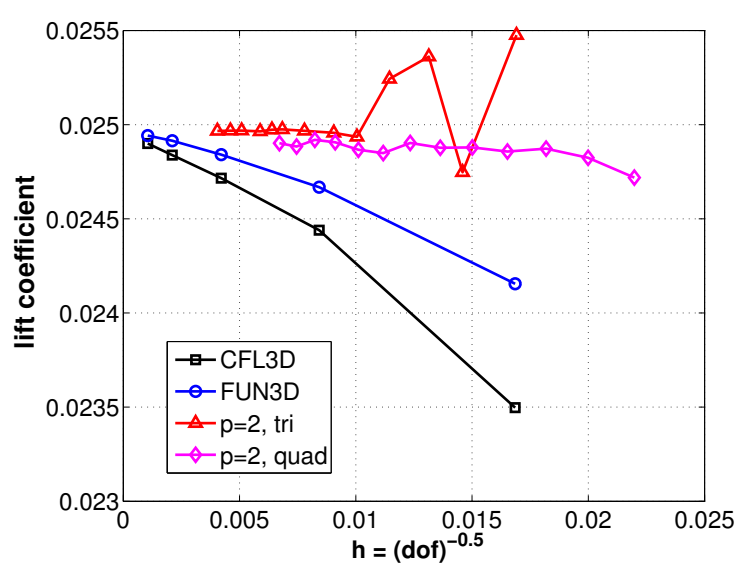

(b) Lift coefficient

Figure 8. Smooth bump: drag and lift coefficient convergence comparisons for drag adaptation with $p=2$, using unstructured (tri) and hanging-node (quad) meshes.

Figures 9 and 10 show selected meshes in the adaptive refinement sequence. As expected, the adaptive refinement targets the boundary layer region, where anisotropic elements are possible, whereas most of the remainder of the flow is approximated with isotropic elements.

VI.C. NACA 0012, $R e=6 \times 10^{6}, M=0.15$

In this case we consider a NACA 0012 airfoil in $R e=6 \times 10^{6}, M=0.15$ flow. The dynamic viscosity is computed using Sutherland's law, Eqn. 2, with $T_{\mathrm{s}}=110 \mathrm{~K}$ and $T_{\text {ref }}=300 \mathrm{~K}$. The inflow turbulence eddy viscosity, $\mu_{t}$ is set to 3 times the laminar viscosity. Free-stream boundary conditions are imposed at a farfield that is over 1500 chords away from the airfoil in each direction.

This case was run adaptively at $p=2$ using hanging-node refinement of a structured initial mesh, with drag as the target output and a fixed refinement fraction of $f^{\text {frac }}=.07$. Figure 11 shows the initial mesh and adapted results for $\alpha=10^{\circ}$. The regions targeted for refinement include the boundary layer, wake, and leading-edge stagnation streamline, where errors can have a large effect on the drag output.

Figure 12 shows a comparison of pressure coefficient and skin friction distributions for $\alpha=0^{\circ}$ and $\alpha=10^{\circ}$. The comparison is made against data from CFL3D, and the results are in excellent agreement: the curves are virtually on top of each other. Figure 13 shows the lift coefficient versus angle of attack and drag polar for the adapted results. Again, excellent agreement with CFL3D data is observed.

Finally, Figure 14 shows the convergence of the lift and drag coefficients with adaptive refinement for two runs: $\alpha=0^{\circ}$ and $\alpha=10^{\circ}$. We see that drag converges faster than lift, which makes sense as we are adapting on the drag outputs. In addition, convergence slows with increasing angle of attack, likely because the flow-field becomes more complex - e.g. the boundary layer on the upper surface becomes thicker and requires more resolution. In all cases, for the last several adaptive iterations, the outputs show little variation. 


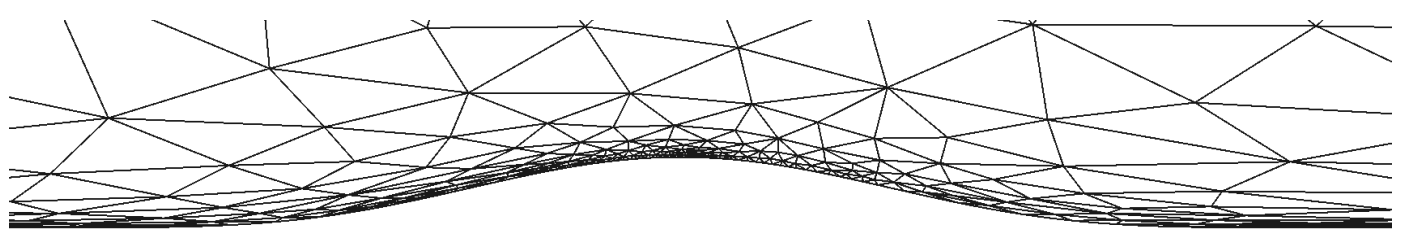

(a) Adaptation iteration 1

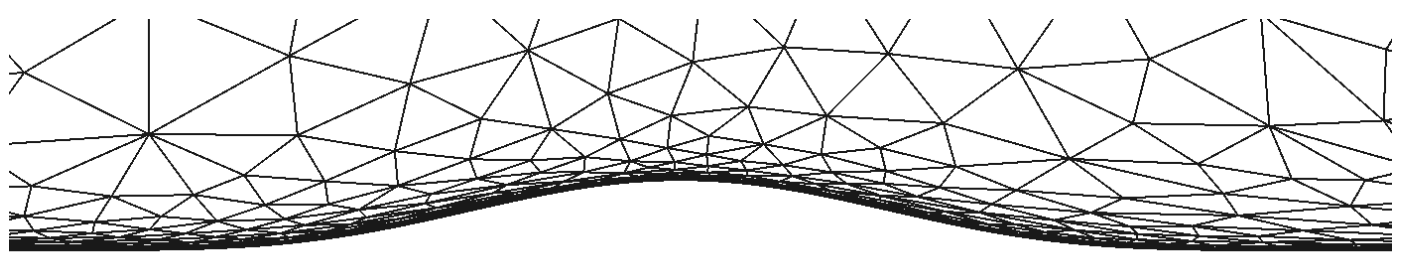

(b) Adaptation iteration 6

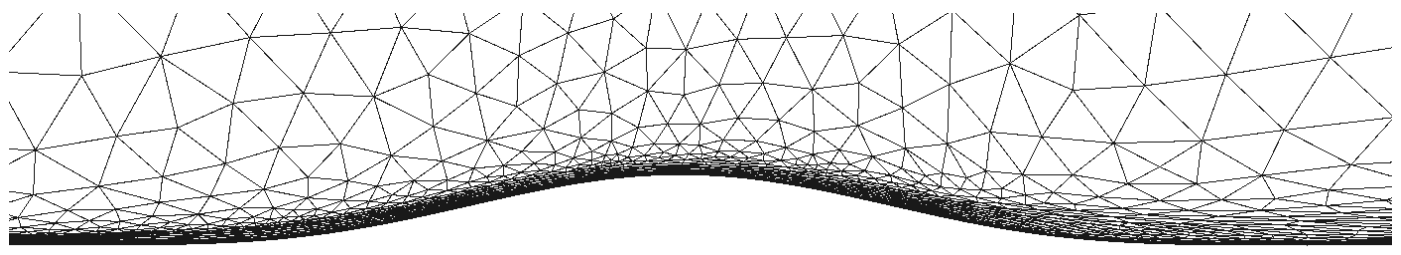

(c) Adaptation iteration 11

Figure 9. Smooth bump: Adapted meshes generated by unstructured metric-based re-meshing and $p=2$ approximation.

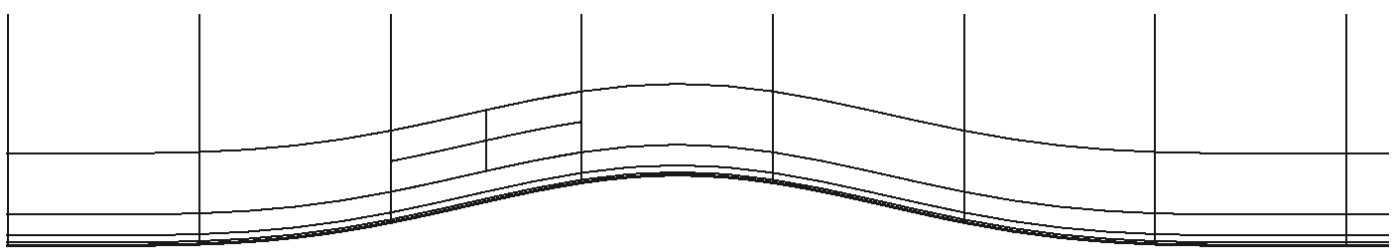

(a) Adaptation iteration 1

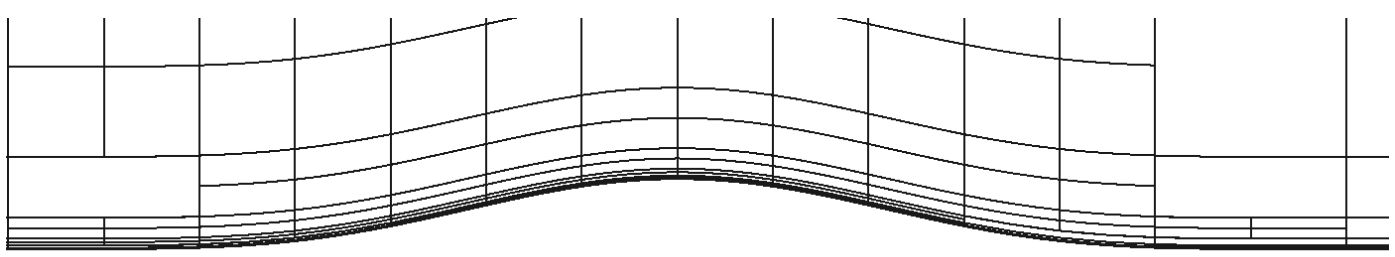

(b) Adaptation iteration 6

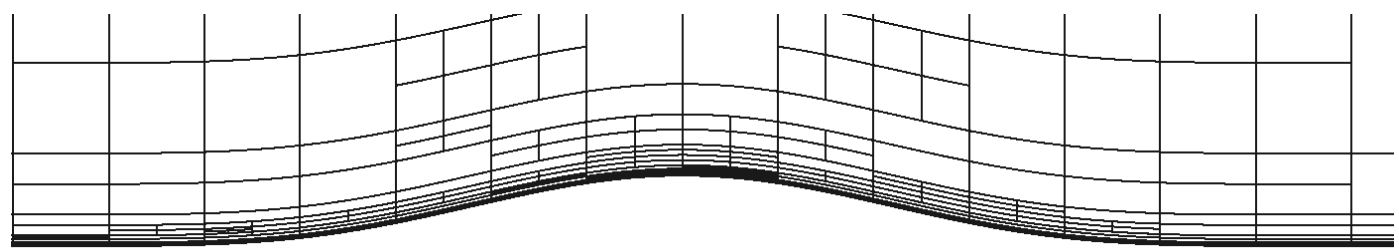

(c) Adaptation iteration 11

Figure 10. Smooth bump: Adapted meshes generated by hanging-node refinement and $p=2$ approximation. 


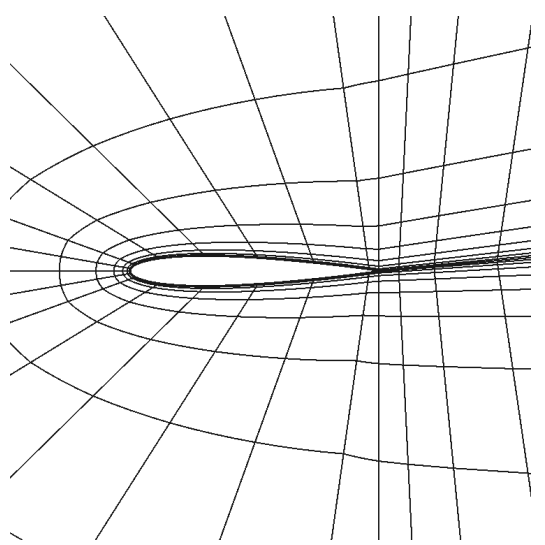

(a) Initial mesh

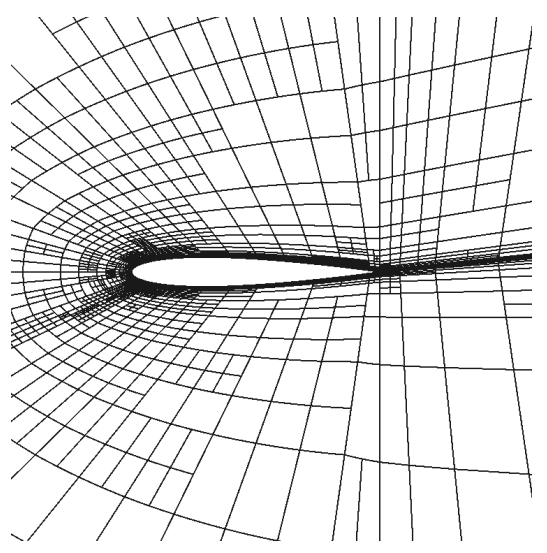

(b) Adapted mesh for $\alpha=10^{\circ}$

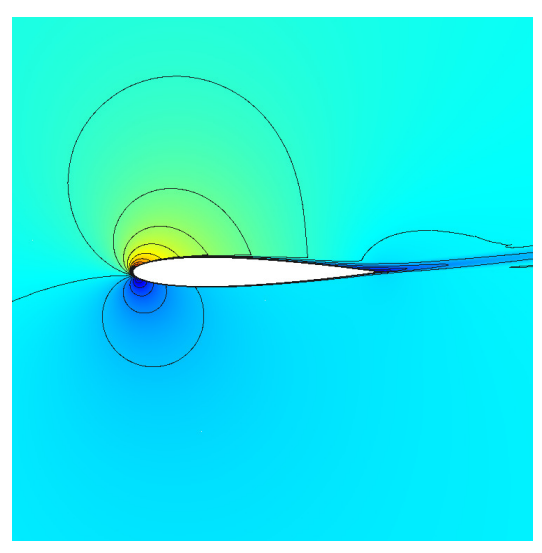

(c) Mach field (0-0.4) for $\alpha=10^{\circ}$

Figure 11. NACA 0012: Initial mesh, drag-adapted mesh, and Mach contours for $\alpha=10^{\circ}$.

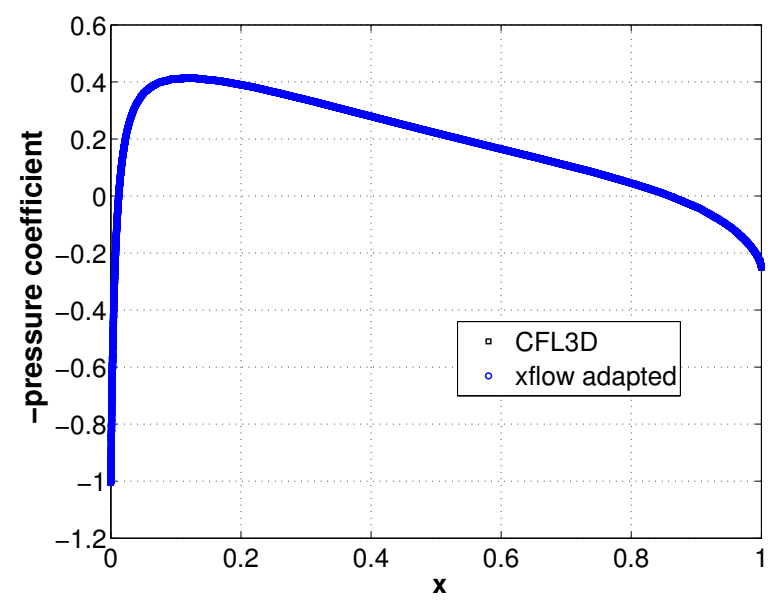

(a) $c_{p}, \alpha=0^{\circ}$

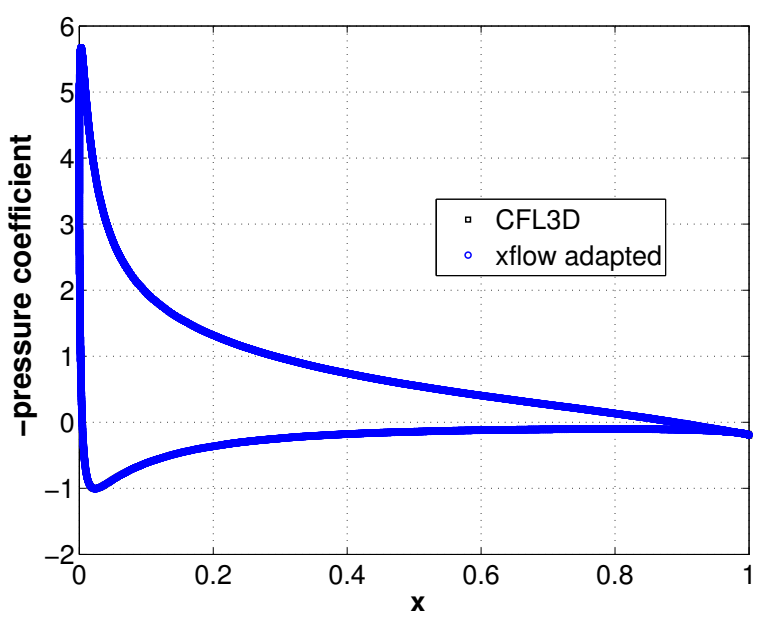

(c) $c_{p}, \alpha=10^{\circ}$

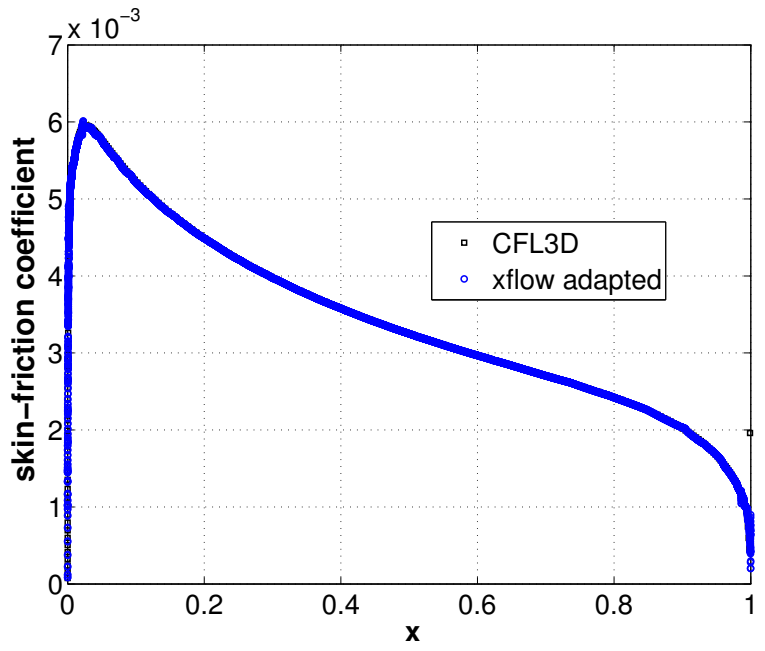

(b) $c_{f}, \alpha=0^{\circ}$

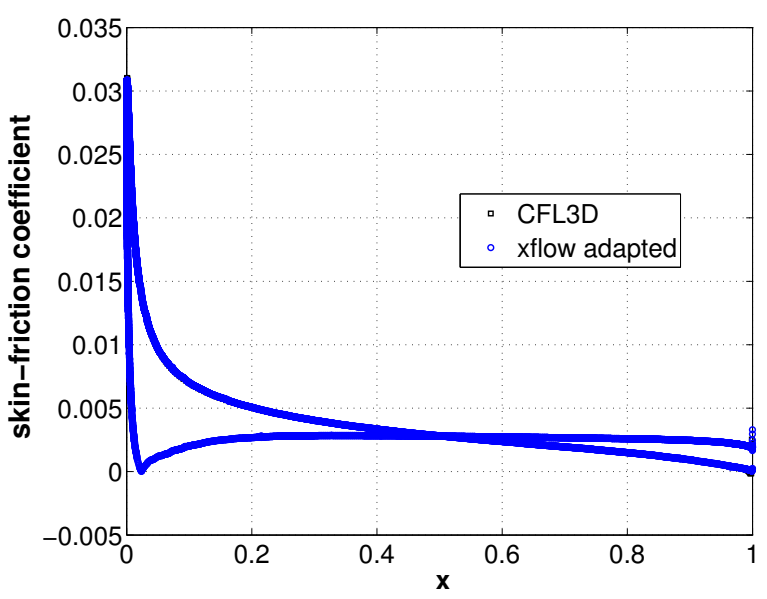

(d) $c_{f}, \alpha=10^{\circ}$

Figure 12. NACA 0012: Pressure and skin-friction coefficient distributions for $\alpha=0,10^{\circ}$, comparing the final adapted mesh result with data from CFL3D. 


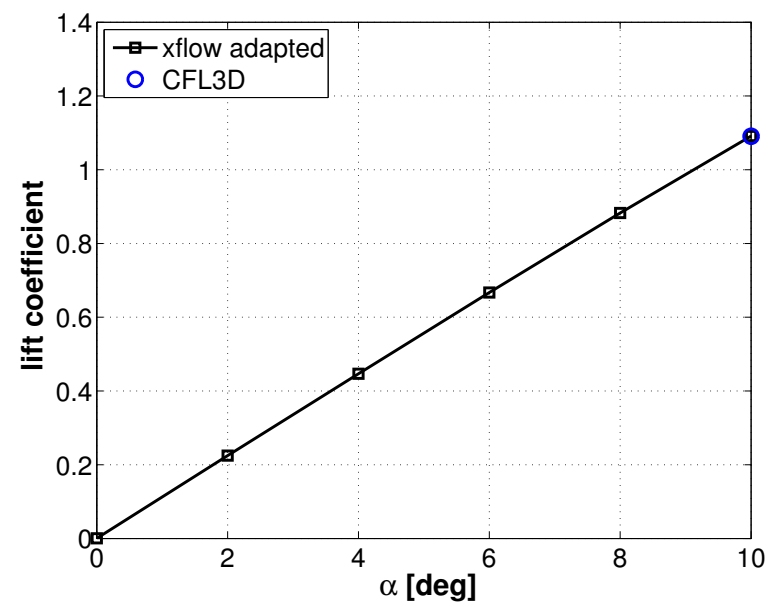

(a) $c_{l}$ versus $\alpha$

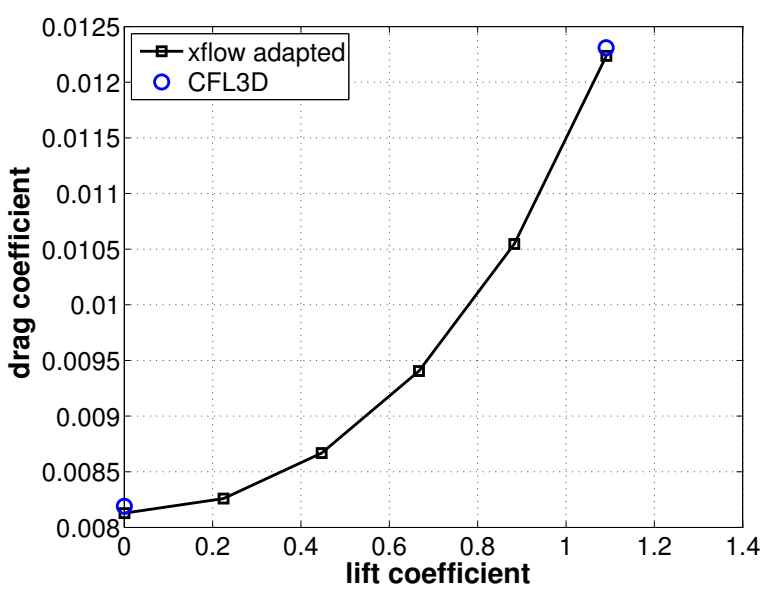

(b) $c_{d}$ versus $c_{l}$

Figure 13. NACA 0012: lift coefficient versus angle of attack and drag polar, with comparison to CFL3D data.

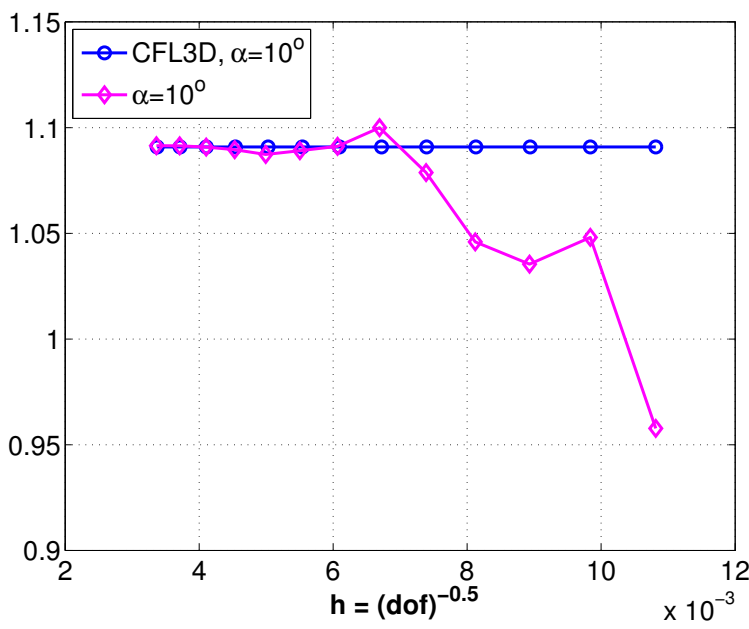

(a) Lift coefficient

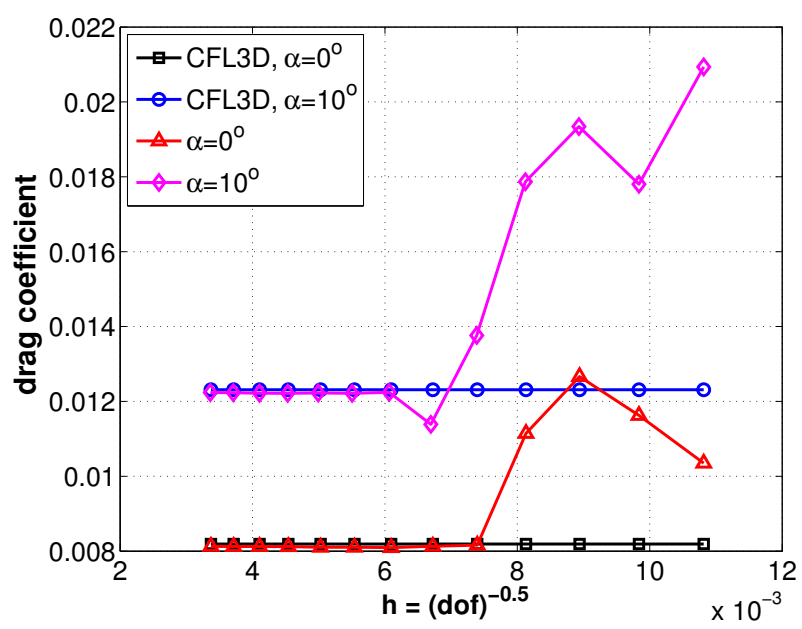

(b) Drag coefficient

Figure 14. NACA 0012: Convergence of lift and drag coefficients with adaptive mesh refinement and $p=2$, for $\alpha=0^{\circ}, 10^{\circ}$. Comparisons with fine-mesh CFL3D data obtained from the NASA turbulence modeling resource group. 
VI.D. NACA 0012, $R e=6 \times 10^{6}, M=0.15, \alpha=10^{\circ}$

In this case we consider a slight variant of the previous test case for the purpose of verification with detailed data made available by the NASA turbulence modeling resource group. The airfoil is still a NACA 0012, with a closed trailing edge as prescribed on the NASA website. The farfield is approximately 500 chords away from the airfoil, but the farfield geometry is constructed to be consistent with the farfield geometry of the grids provided on the NASA website. No vortex correction is employed on the farfield. The website also provides detailed conditions and setup information on the case.

This case was run adaptively at $p=2$ using metric-based triangular refinement of a relatively coarse initial mesh. Drag is chosen as the target output and a fixed growth fraction of $f^{\text {growth }}=1.3$ is used. Figure 15 shows the initial mesh and adapted results. The regions targeted for refinement include the boundary layer, wake, and leading-edge stagnation streamline. Figure 16 shows a close-up of the leading and trailing edge regions for the $12^{\text {th }}$ adapted mesh.

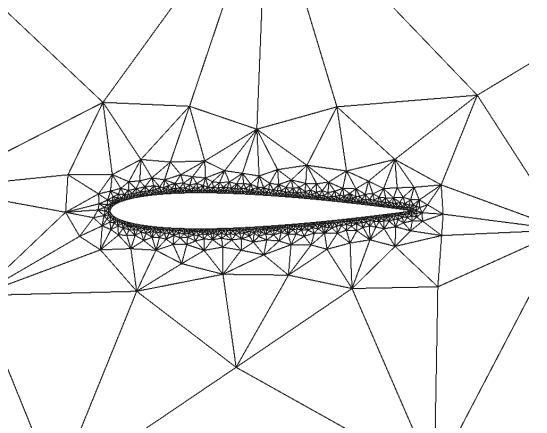

(a) Initial mesh

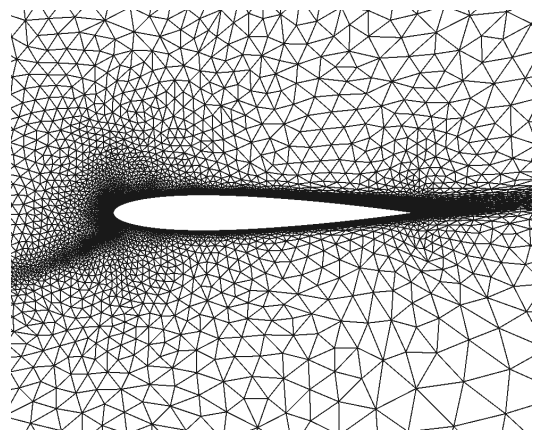

(b) Adapted mesh

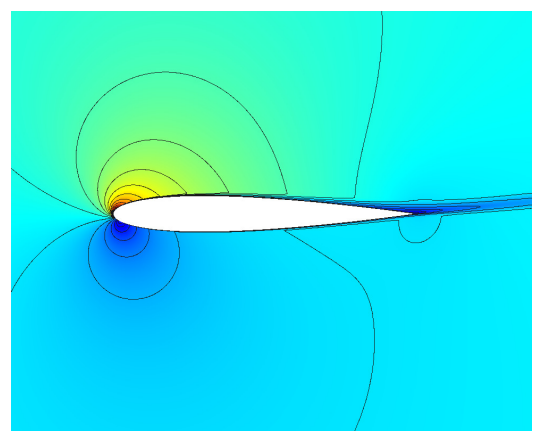

(c) Mach field (0-0.4)

Figure 15. NACA 0012, $\alpha=10^{\circ}$ : Initial mesh, drag-adapted mesh, and Mach contours.

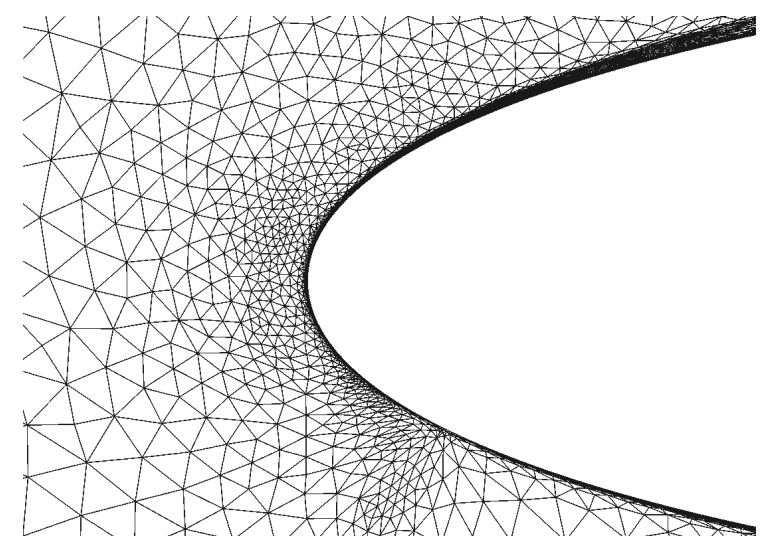

(a) Leading-edge zoom

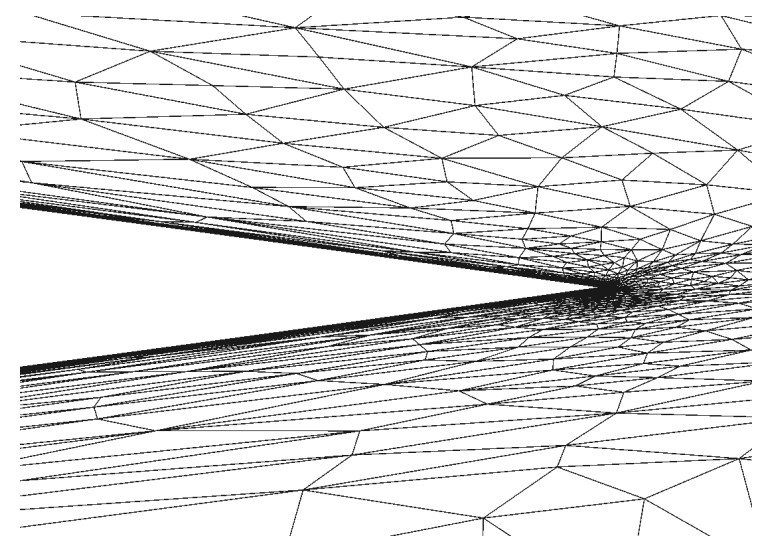

(b) Trailing edge zoom

Figure 16. NACA $0012, \alpha=10^{\circ}$ : Close-up of the leading and trailing edges for the $12^{\text {th }}$ adapted mesh in a drag refinement sequence.

Figure 17 shows the convergence of the lift and drag coefficients with adaptive refinement. We see that both coefficients agree well with the provided data obtained from the CFL3D and FUN3D codes. The adapted values still show variation on the finest grids, and this variation could be due to insufficient resolution (i.e. more adaptations needed) or to an inadequate measure of anisotropy (currently based on the Mach Hessian) during mesh optimization. Future work will investigate the precise cause and possible mesh efficiency improvements. 


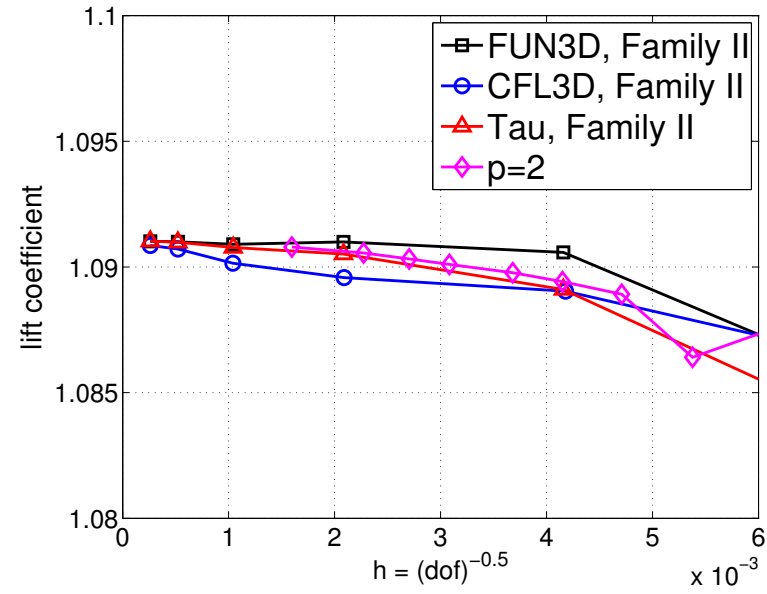

(a) Lift coefficient

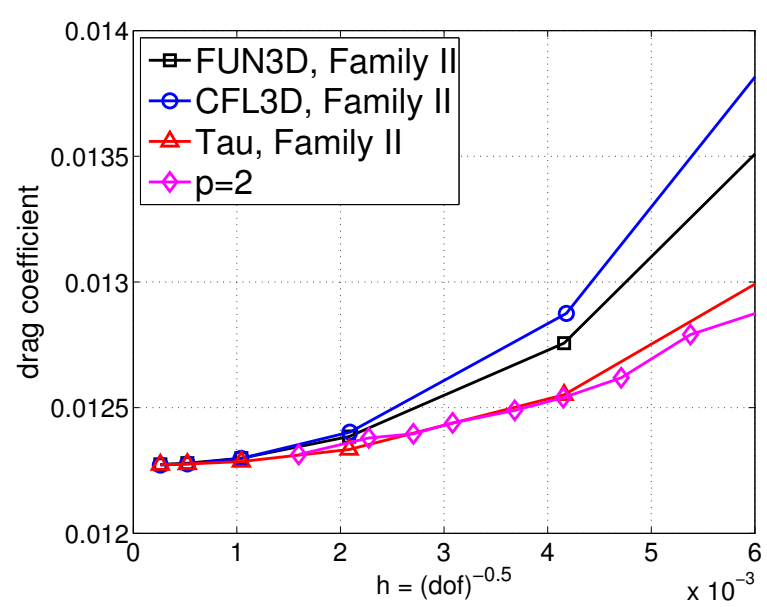

(b) Drag coefficient

Figure 17. NACA 0012, $\alpha=10^{\circ}$ : Convergence of lift and drag coefficients with adaptive mesh refinement and $p=2$, compared with FUN3D and CFL3D data obtained from the NASA turbulence modeling resource group.

Finally, Figure 18 shows the pressure coefficient off the bottom surface of the airfoil trailing edge at $x=0.999 c$. This pressure coefficient was obtained from a $p=3$ run on the finest adapted $p=2$ mesh. Data from the FUN3D on a sequence of grids are overlaid. As shown, the pressure coefficient is close to the fine grid data. It is possible that the drag-adapted mesh, even at an increased approximation order, is not ideally-suited for predicting the off-body pressure coefficient distribution. Adapting on the lift, which would be more sensitive to such pressure errors, could give even better results.

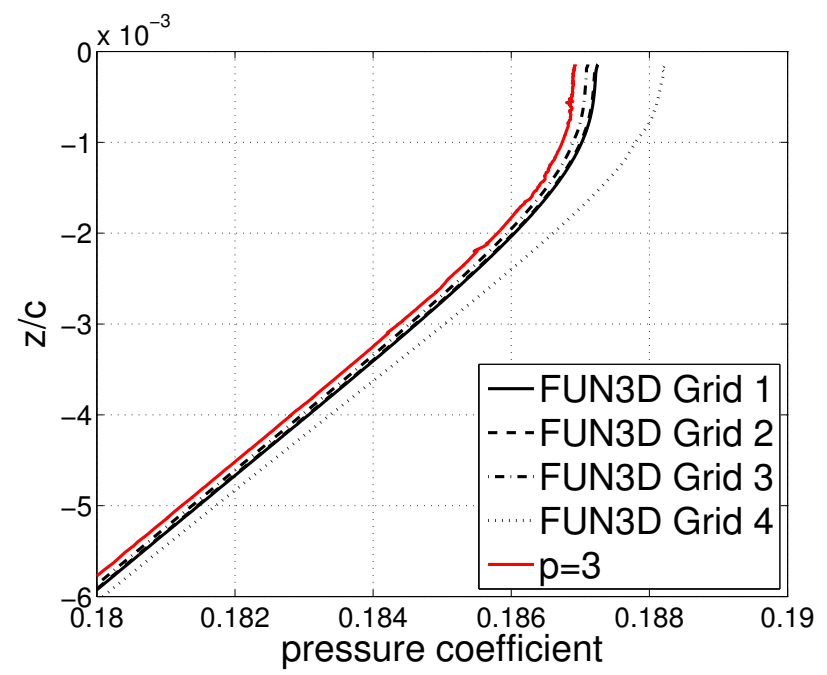

Figure 18. NACA 0012, $\alpha=10^{\circ}$ : Pressure coefficient profiles off the bottom surface of the trailing edge, at $x=0.999 c$.

VI.E. $\quad$ NACA 4412, $R e=1.52 \times 10^{6}, M=0.09, \alpha=13.87^{\circ}$

This test case consists of a NACA 4412 airfoil at high angle of attack, $\alpha=13.87^{\circ}$, at $R e=1.52 \times 10^{6}$, $M=0.09$. The dynamic viscosity is computed using Sutherland's law, Eqn. 2, with $T_{\mathrm{s}}=110 \mathrm{~K}$ and $T_{\text {ref }}=297.8 \mathrm{~K}$. The inflow turbulence eddy viscosity, $\mu_{t}$ is set to 3 times the laminar viscosity. Free-stream boundary conditions are imposed at a farfield that is over 1500 chords away from the 
airfoil in each direction.

This case was run adaptively using hanging-node refinement of a structured initial mesh, with drag as the target output and a fixed refinement fraction of $f^{\text {frac }}=.07$. Figure 19 shows the Mach number contours, an adapted mesh, wall distance contours, and the $x$-momentum component of the drag adjoint. The regions targeted for refinement include the boundary layer and wake, but also a large portion of the mesh in front of the airfoil, on the leading-edge stagnation streamline - note that the adjoint exhibits rapid variation in this area, indicating that error sources near the stagnation streamline can have a large effect on the drag output.

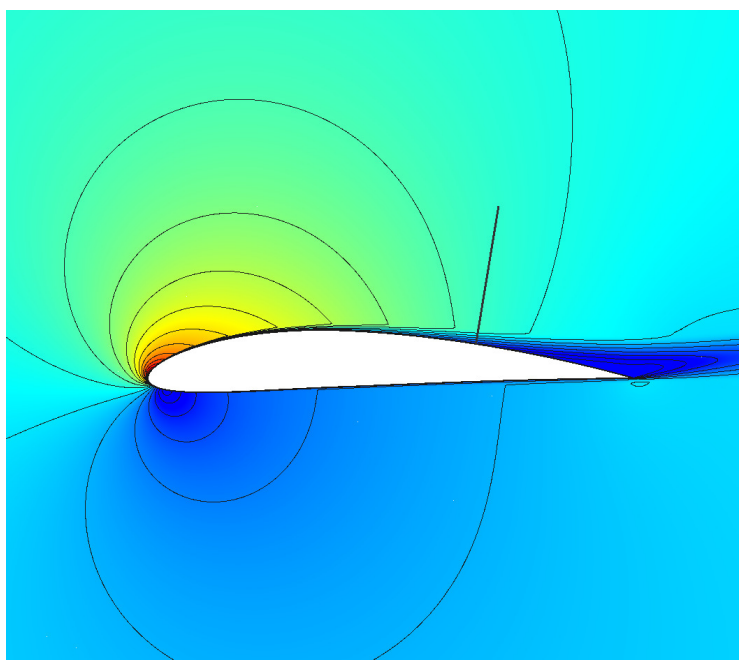

(a) Mach contours $(0-0.25)$ and line probe

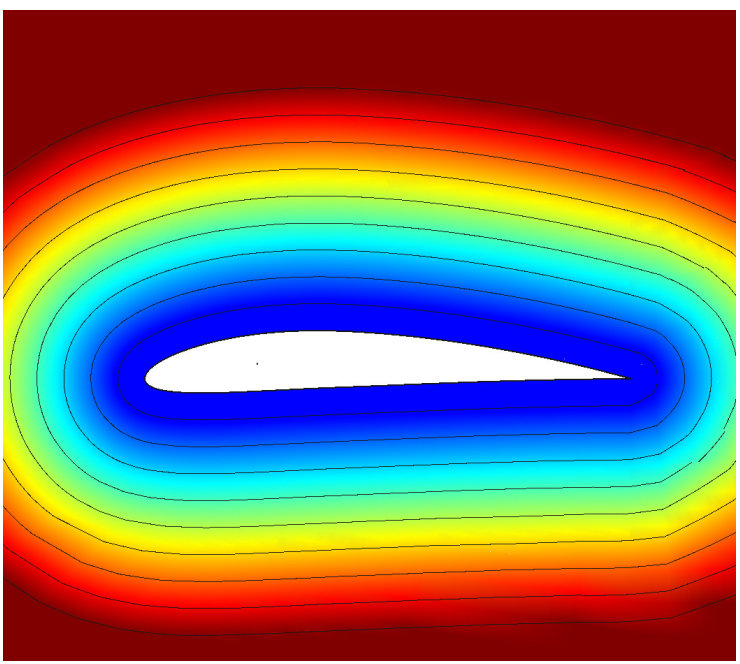

(c) Wall distance contours (0-0.5)

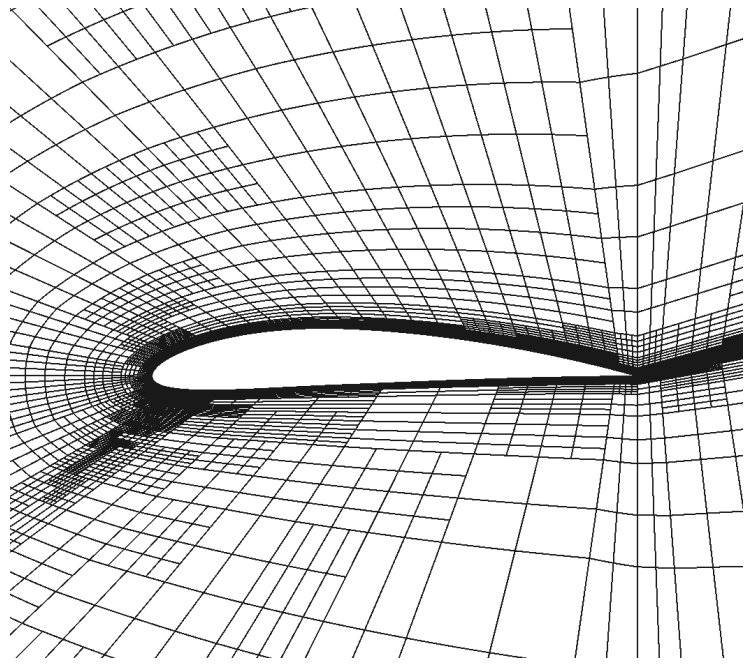

(b) Adapted mesh

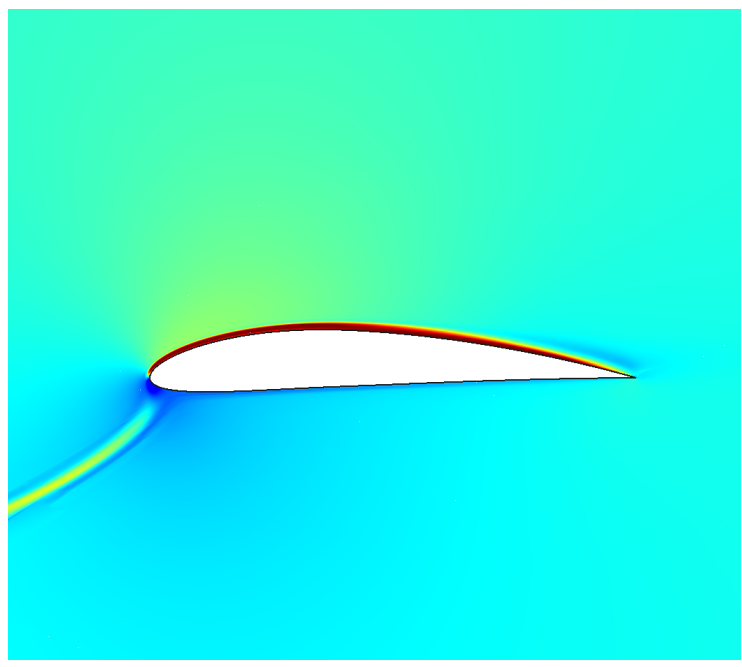

(d) $x$-Momentum drag adjoint

Figure 19. NACA 4412: Mesh and field plots from adaptive simulation results.

Figure 20 shows velocity profiles along line probes extending roughly normal to the wall at several locations on the aft portion of the airfoil upper surface. The mesh used for this comparison was the final $p=2$ adapted mesh (after 10 adaptive iterations), uniformly refined and with order increased to $p=3$ (a total of 140880 degrees of freedom). Experimental data are available at points on these lines, as are data from other codes, including CFL3D; in this work, we focus on code-tocode verification. Compared with CFL3D the horizontal and vertical velocities along the lines are very close. The agreement is not exact, and the small differences could be due to variants in the 
SA turbulence model or to numerical errors. Note that while our data came from meshes adapted to reduce the numerical error, only numerical errors affecting the drag output were targeted, so that the velocity profiles could potentially not yet be converged.

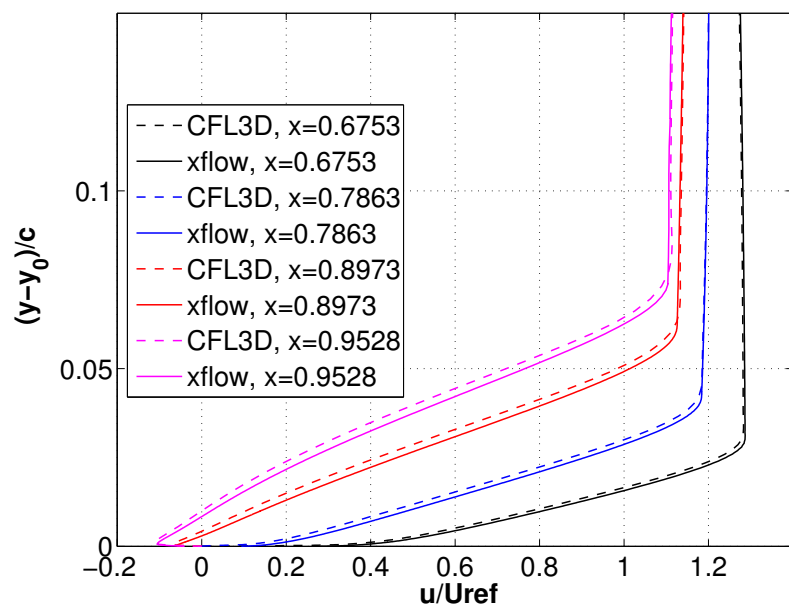

(a) Horizontal velocity profiles

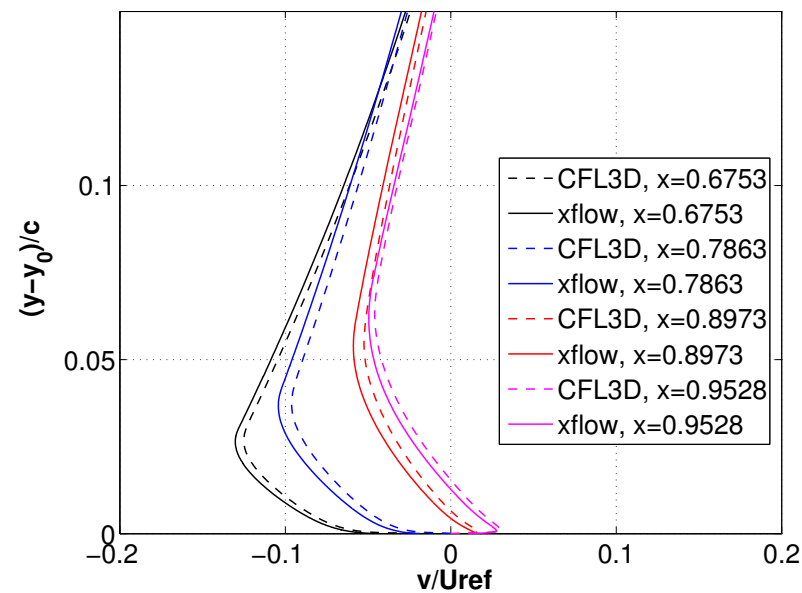

(b) Vertical velocity profiles

Figure 20. NACA 4412: Velocity profile comparisons for several wall-normal line probes on the aft portion of the airfoil upper surface. "xflow" (the present code) data are obtained from a simulation on the final adapted $p=2$ mesh, uniformly refined and at order $p=3$. CFL3D data are obtained from the NASA turbulence modeling resource group.

Figure 21 shows the convergence of the drag and lift coefficients with adaptive refinement. Though we only adapt on drag, lift is a similar output and exhibits good convergence too. We see that the drag converges rapidly with indiscernible variations past $h=.01$, or 10000 degrees of freedom for both $p=1$ and $p=2$ approximation. The lift takes a little longer, in part because of higher sensitivity of the lift to refinement at the trailing edge and because we do not specifically target the lift. For the drag, since we use an adjoint-based method, we have an output error estimate (the adjoint-weighted residual) at each adaptive iteration. We can use this error estimate to correct the drag - these corrected outputs are also shown in Figure 21. As we converge the adjoint solution to high precision on the fine space, we obtain excellent error corrections: even with $p=1$ approximation, the corrected drag varies little after about 5000 degrees of freedom.

\section{Conclusions}

We present a high-order output-based adaptive solution technique for the RANS equations closed with a recent variant of the Spalart-Allmaras model, "SA-neg". We use discontinuous finite elements for the discretization and present key practical details relevant to the implementation. The results compare two variants of the high-order adaptive solution technique to each other and to standard second-order techniques in terms of accuracy versus degrees-of-freedom. Isotropic quadrilateral-element adaptation using hanging-nodes and an anisotropic initial mesh is found to yield similar asymptotic results compared to metric-based unstructured mesh refinement. Using the easy-to-generate isotropic unstructured initial meshes considered, the unstructured adaptation produced larger errors on the first adaptive iterations compared to hanging-node refinement with a more meticulously tailored initial structured mesh. This result is expected given the higher-quality of the initial structured meshes. The ability of the unstructured method to automatically "snap" to the RANS mesh from an initially-isotropic mesh is a desirable capability, though it requires robustness of the solver to under-resolution. 


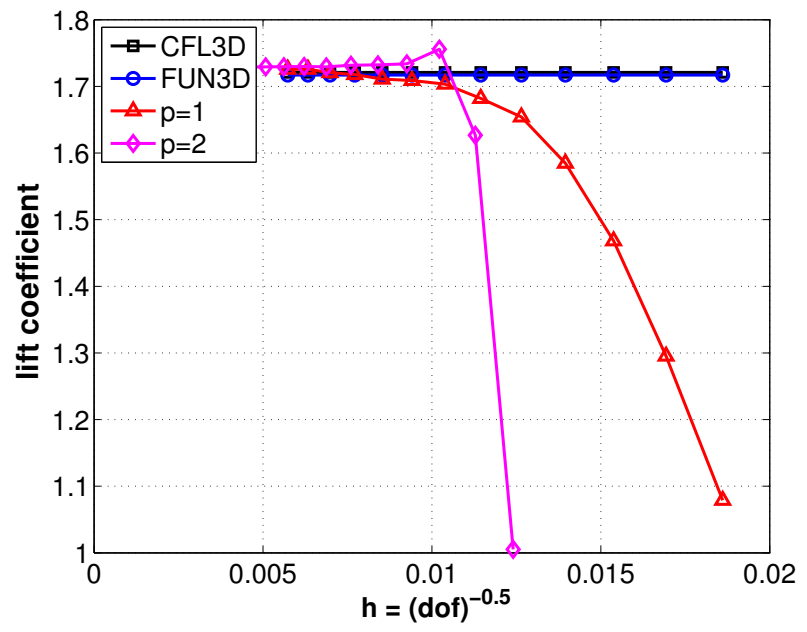

(a) Lift coefficient

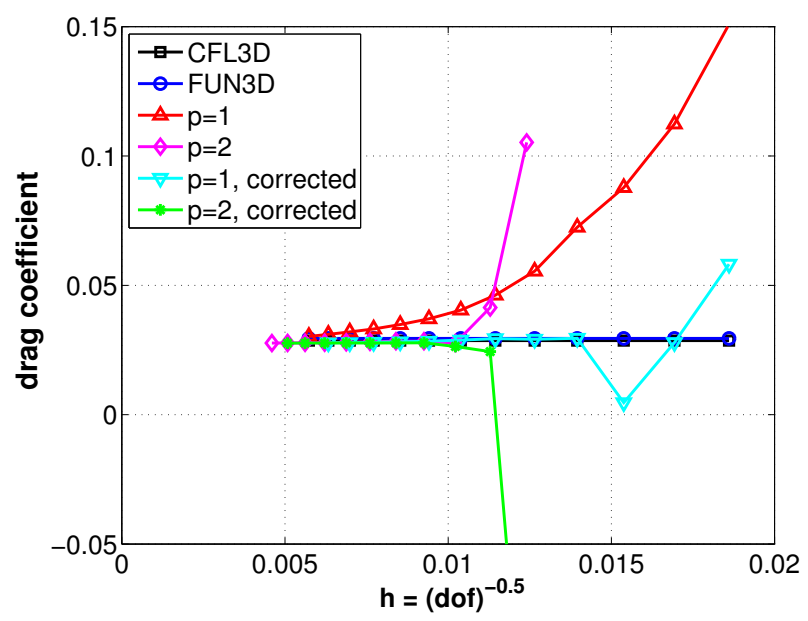

(b) Drag coefficient

Figure 21. NACA 4412: Convergence of lift and drag coefficients with adaptive mesh refinement for $p=1$ and $p=2$. Note, "corrected" results refer to subtracting the adjoint-weighted residual error estimate from the measured output. Comparisons with fine-mesh CFL3D and FUN3D data obtained from the NASA turbulence modeling resource group.

Relative to uniform refinement at second order, high-order adaptation is found to yield faster convergence - the adaptive runs often quickly snap (close) to the correct solution in a few steps. This comparison does not take into account the computational cost of the error estimation and adaptation, which is primarily that of the fine-space adjoint solve. However, even though the fine space involves an order increment, because the adjoint problem is linear whereas the RANS equations are highly non-linear, the adjoint cost is less than (at most $\sim 25 \%$ ) that of the primal for all cases considered. A topic for future studies, however, is a reduction in the cost of the primal solve, which for our implementation is likely larger than that of the second-order methods for a given error level.

\section{Acknowledgments}

The authors acknowledge support from the Air Force Office of Scientific Research under grant FA9550-11-1-0081.

\section{References}

${ }^{1}$ Yano, M., Modisette, J., and Darmofal, D., "The Importance of mesh adaptation for higher-order discretizations of aerodynamics flows," AIAA Paper 2011-3852, 2011.

${ }^{2}$ Becker, R. and Rannacher, R., "An optimal control approach to a posteriori error estimation in finite element methods," Acta Numerica, edited by A. Iserles, Cambridge University Press, 2001, pp. 1-102.

${ }^{3}$ Venditti, D. A. and Darmofal, D. L., "Anisotropic grid adaptation for functional outputs: application to two-dimensional viscous flows," Journal of Computational Physics, Vol. 187, No. 1, 2003, pp. 22-46.

${ }^{4}$ Hartmann, R. and Houston, P., "Adaptive discontinuous Galerkin finite element methods for the compressible Euler equations," Journal of Computational Physics, Vol. 183, No. 2, 2002, pp. 508-532.

${ }^{5}$ Fidkowski, K. J. and Darmofal, D. L., "Review of Output-Based Error Estimation and Mesh Adaptation in Computational Fluid Dynamics," American Institute of Aeronautics and Astronautics Journal, Vol. 49, No. 4, 2011, pp. 673-694.

${ }^{6}$ Allmaras, S., Johnson, F., and Spalart, P., "Modifications and Clarifications for the Implementation of the Spalart-Allmaras Turbulence Model," Seventh International Conference on Computational Fluid Dynamics (ICCFD7) 1902, 2012. 
${ }^{7}$ Oliver, T. A., A High-order, Adaptive, Discontinuous Galerkin Finite Elemenet Method for the ReynoldsAveraged Navier-Stokes Equations, Ph.D. thesis, Massachusetts Institute of Technology, Cambridge, Massachusetts, 2008.

${ }^{8}$ Oliver, T. A. and Darmofal, D. L., "Impact of Turbulence Model Irregularity on High-order Discretizations," AIAA Paper 2009-953, 2009.

${ }^{9}$ Ceze, M. A. and Fidkowski, K. J., "An anisotropic hp-adaptation framework for functional prediction," American Institute of Aeronautics and Astronautics Journal, Vol. 51, 2013, pp. 492-509.

${ }^{10}$ Yano, M., An Optimization Framework for Adaptive Higher-Order Discretizations of Partial Differential Equations on Anisotropic Simplex Meshes, Ph.D. thesis, Massachusetts Institute of Technology, Cambridge, Massachusetts, 2012.

${ }^{11}$ Ceze, M. A. and Fidkowski, K. J., "Drag Prediction Using Adaptive Discontinuous Finite Elements," $A I A A$ Journal of Aircraft, Vol. 51, No. 4, 2014, pp. 1284-1294.

${ }^{12}$ Roe, P. L., "Approximate Riemann solvers, parameter vectors, and difference schemes," Journal of Computational Physics, Vol. 43, 1981, pp. 357-372.

${ }^{13}$ Bassi, F. and Rebay, S., "Numerical evaluation of two discontinuous Galerkin methods for the compressible Navier-Stokes equations," International Journal for Numerical Methods in Fluids, Vol. 40, 2002, pp. $197-207$.

${ }^{14}$ Oliver, T. and Darmofal, D., "Analysis of dual consistency for discontinuous Galerkin discretizations of source terms." SIAM Journal of Numerical Analysis, Vol. 47, 2009, pp. 3507-3525.

${ }^{15}$ Leicht, T. and Hartmann, R., "Error estimation and anisotropic mesh refinement for 3d laminar aerodynamic flow simulations," Journal of Computational Physics, Vol. 229, 2010, pp. 7344-7360.

${ }^{16}$ Ceze, M. and Fidkowski, K. J., "Pseudo-transient Continuation, Solution Update Methods, and CFL Strategies for DG Discretizations of the RANS-SA Equations," AIAA Paper 2013-2686, 2013.

${ }^{17}$ Fidkowski, K. J., Oliver, T. A., Lu, J., and Darmofal, D. L., "p-Multigrid solution of high-order discontinuous Galerkin discretizations of the compressible Navier-Stokes equations," Journal of Computational Physics, Vol. 207, 2005, pp. 92-113.

${ }^{18}$ Ceze, M. A. and Fidkowski, K. J., "Output-Driven Anisotropic Mesh Adaptation for Viscous Flows Using Discrete Choice Optimization," AIAA Paper 2010-0170, 2010.

${ }^{19}$ Fidkowski, K. J. and Darmofal, D. L., "A triangular cut-cell adaptive method for high-order discretizations of the compressible Navier-Stokes equations," Journal of Computational Physics, Vol. 225, 2007, pp. $1653-1672$.

${ }^{20}$ Borouchaki, H., George, P., Hecht, F., Laug, P., and Saltel, E., "Mailleur bidimensionnel de Delaunay gouverné par une carte de métriques. Partie I: Algorithmes," INRIA-Rocquencourt, France. Tech Report No. $2741,1995$.

${ }^{21}$ Baker, T. J., "Mesh Adaptation Strategies for Problems in Fluid Dynamics," Finite Elements in Analysis and Design, Vol. 25, 1997, pp. 243-273.

${ }^{22}$ Castro-Diaz, M. J., Hecht, F., Mohammadi, B., and Pironneau, O., "Anisotropic unstructured mesh adaptation for flow simulations," International Journal for Numerical Methods in Fluids, Vol. 25, 1997, pp. 475-491. 